\title{
A CARP-1 functional mimetic compound is synergistic with BRAF-targeting in non-small cell lung cancers
}

\author{
Vino T. Cheriyan ${ }^{1,2}$, Hashem Alsaab ${ }^{5,7}$, Sreeja Sekhar ${ }^{1,2}$, Jaganathan Venkatesh ${ }^{1,2}$, \\ Arindam Mondal ${ }^{6}$, Imran Vhora ${ }^{6}$, Samaresh Sau ${ }^{5}$, Magesh Muthu ${ }^{1,2,8}$, Lisa A. Polin ${ }^{2,4}$, \\ Edi Levi ${ }^{1,3}$, Gerold Bepler ${ }^{2}$, Arun K. Iyer ${ }^{4,5}$, Mandip Singh ${ }^{6}$ and Arun K. Rishi ${ }^{1,2,4}$ \\ ${ }^{1}$ John D. Dingell VA Medical Center, Detroit, MI, 48201, USA \\ ${ }^{2}$ Department of Oncology, Barbara Ann Karmanos Cancer Institute, Wayne State University, School of Medicine, Detroit, MI, \\ 48201, USA \\ ${ }^{3}$ Department of Pathology, Wayne State University, School of Medicine, Detroit, MI, 48201, USA \\ ${ }^{4}$ Molecular Therapeutics Program, Barbara Ann Karmanos Cancer Institute, Wayne State University, School of Medicine, \\ Detroit, MI, 48201, USA \\ ${ }^{5}$ Use-inspired Biomaterials and Integrated Nano Delivery (U-BiND) Systems Laboratory Department of Pharmaceutical \\ Sciences, Eugene Applebaum College of Pharmacy and Health Sciences, Wayne State University, Detroit, MI 48201, USA \\ ${ }^{6}$ College of Pharmacy and Pharmaceutical Sciences, Florida A\&M University, Tallahassee, FL 32307, USA \\ ${ }^{7}$ Department of Pharmaceutics and Pharmaceutical Technology, College of Pharmacy, Taif University, Taif 26571, Saudi Arabia \\ ${ }^{8}$ Present Address: Department of Molecular Biology, Umeå University, Umeå 90187, Sweden
}

Correspondence to: Arun K. Rishi, email: rishia@Karmanos.org

Keywords: NSCLC; TKIs; CFM; CARP-1/CCAR1; B-Raf-inhibitors

Received: January 03, $2018 \quad$ Accepted: May 15, $2018 \quad$ Published: July 03, 2018

Copyright: Cheriyan et al. This is an open-access article distributed under the terms of the Creative Commons Attribution License 3.0 (CC BY 3.0), which permits unrestricted use, distribution, and reproduction in any medium, provided the original author and source are credited.

\section{ABSTRACT}

Non-small cell lung cancers (NSCLC) account for $85 \%$ of all lung cancers, and the epidermal growth factor receptor (EGFR) is highly expressed or activated in many NSCLC that permit use of EGFR tyrosine kinase inhibitors (TKIs) as frontline therapies. Resistance to EGFR TKIs eventually develops that necessitates development of improved and effective therapeutics. CARP-1/CCAR1 is an effector of apoptosis by Doxorubicin, Etoposide, or Gefitinib, while CARP-1 functional mimetic (CFM) compounds bind with CARP-1, and stimulate CARP-1 expression and apoptosis. To test whether CFMs would inhibit TKI-resistant NSCLCs, we first generated and characterized TKI-resistant NSCLC cells. The $\mathbf{G I}_{50}$ dose of Erlotinib for parental and Erlotinib-resistant HCC827 cells was $\sim 0.1 \mu \mathrm{M}$ and $\geq 15 \mu \mathrm{M}$, respectively. While Rociletinib or Ocimertinib inhibited the parental $\mathrm{H} 1975$ cells with $\mathrm{GI}_{50}$ doses of $\leq 0.18$ $\mu \mathrm{M}$, the Ocimertinib-resistant pools of $\mathrm{H1975}$ cells had a $\mathrm{GI}_{50}$ dose of $\sim 12 \mu \mathrm{M}$. The $\mathbf{G I}_{50}$ dose for Rociletinib-resistant $\mathrm{H1975}$ sublines ranged from 4.5-8.0 $\mu \mathrm{M}$. CFM-4 and its novel analog CFM-4.16 attenuated growth of the parental and TKI-resistant NSCLC cells. CFMs activated p38/JNKs, inhibited oncogenic cMet and Akt kinases, while CARP-1 depletion blocked NSCLC cell growth inhibition by CFM-4.16 or Erlotinib. CFM4.16 was synergistic with B-Raf-targeting in NSCLC, triple-negative breast cancer, and renal cancer cells. A nano-lipid formulation (NLF) of CFM-4.16 in combination with Sorafenib elicited a superior growth inhibition of xenografted tumors derived from Rociletinib-resistant H1975 NSCLC cells in part by stimulating CARP-1 and apoptosis. These findings support therapeutic potential of CFM-4.16 together with B-Raf targeting in treatment of TKI-resistant NSCLCs. 


\section{INTRODUCTION}

Lung carcinoma is the leading cause of cancer death in not only the United States but worldwide [1-2]. With current treatments, non-small cell carcinoma (NSCLC), which accounts for approximately $85 \%$ of lung cancer cases, carries a 5 year survival of $14 \%$ for all stages [13]. Oncogenic epidermal growth factor receptor (EGFR) tyrosine kinase (RTK) is a driver of a significant subset of NSCLCs and is an indicator of poor prognosis. The EGFR-driven NSCLCs are unresponsive to the frontline chemotherapeutic Cisplatin. Accordingly, EGFR TKIs are widely used to treat EGFR-driven NSCLCs. The first and second generation TKIs target the enzyme activity of EGFR, and thus inhibit NSCLC cell growth and survival signaling. Interestingly, a significant subset of NSCLCs harbor classical activating mutation in the kinase domain of the EGFR [2, 4]. This deletion of EGFR exon 19 $(\Delta 19)$ is associated with good clinical responses to first generation EGFR TKIs such as Gefitinib or Erlotinib $[2,4]$. However, a vast majority of patients develop resistance to these TKIs due in part to activation and/or expression of alternate, redundant RTKs as well as emergence of the "gatekeeper" T790M mutation in the kinase domain of EGFR. This EGFR T790M mutation leads to resistance to most clinically available first and second generation EGFR TKIs by increasing the affinity of the receptor to adenosine triphosphate (ATP). Recently, third generation of EGFR TKIs that are non-ATP-competitive, allosteric inhibitors of mutant EGFR were developed. Following rigorous clinical testing of Rociletinib and Ocimertinib, US FDA approved Ocimertinib for clinical use. However, a number of recent pre-clinical laboratory and animal studies have investigated molecular mechanisms of emergence of NSCLC resistance to these third-generation TKIs $[5,6]$. Thus, there is an urgent need to identify and devise new approaches to treat NSCLCs and their TKIresistant phenotypes.

CARP-1 (Cell cycle and apoptosis regulator 1/ CCAR1), a peri-nuclear phospho-protein, is a regulator of cell growth and apoptosis signaling [7-10]. CARP1 has been previously shown to regulate adipogenesis by functioning as transcriptional co-activator of the steroid receptor, glucocorticoid receptor (GR). CARP-1 also regulates Adriamycin (ADR) dependent apoptosis, mediated in part through p53 co-activation. Thus, CARP-1 co-ordinates both cell growth and apoptosis, functioning as a biphasic regulator $[11,12]$. CARP-1 is often overexpressed in cells experiencing stress induced by withdrawal of growth factors or chemotherapyinduced cell cycle arrest and apoptosis [7, 8, 11]. CARP1 is also known to co-activate the E3 ligase, APC/C, which is involved in cell cycle transitions and also tumor progression. [9, 13-15]. On the basis of CARP-1 coactivation of $\mathrm{APC} / \mathrm{C}$, our recent studies further reported identification and testing of novel, small molecule inhibitors (SMIs) of CARP-1 binding with APC/C subunit APC2 [9]. These compounds, termed CARP1 functional mimetics (CFMs), inhibit cell growth by inducing apoptosis in various cancer cell types $[9,10]$. Genetic studies previously revealed that C.elegans CARP1 homolog lst 3 functioned as an antagonist of EGFR signaling but an agonist of Notch signaling [16], while targeting of EGFR caused CARP-1 increase and apoptosis [8]. We have previously observed increased resistance to apoptosis induced by chemotherapeutic drugs including ADR, Etoposide, CFMs, or EGFR TKI Gefitinib in cells where CARP-1 was knocked down, implicating its critical role in growth inhibition by these agents $[7,8,11]$.

Given that EGFR TKIs remain frontline therapies for a large subset of NSCLCs, and emergence of resistance to TKIs continues to be a significant and unmet challenge, we investigated (a) whether CFM compounds inhibit NSCLC cell growth and (b) the molecular mechanisms by which CFMs inhibit growth of NSCLC cells. In addition, we investigated whether CFMs will also inhibit growth of TKIresistant NSCLC cells. To this end, we first generated and characterized laboratory models of NSCLC cells that harbor mutant EGFR and are resistant to Erlotinib, Rociletinib, or Ocimertinib. Our studies revealed that CFM compound 4.16 inhibited growth of parental and also the TKI-resistant NSCLC cells when used as a single agent. CFM-4.16 synergized with B-Raf-targeting therapies (Sorafenib or Dabrafenib) in vitro. Interestingly. we also observed superior inhibition of Rociletinib-resistant NSCLC cell derived tumor xenografts in immunocompromised mice when treated with a combination of CFM-4.16 nano-lipid formulation and Sorafenib.

\section{RESULTS}

\section{CFM compounds inhibit growth of NSCLC cells}

We have previously reported that CFM compounds possess anti-cancer properties $[9,17-20]$, and we further observed that the CFM-4.16 analog effectively inhibited the growth of parental as well as drug resistant Renal cell carcinoma (RCC), and human and murine triple negative breast cancer (TNBC) cells in vitro and also in vivo. [21, 22]. Given that development of drug-resistant NSCLCs remain a formidable problem that contributes to treatment failure and poor prognosis [2-6], we tested whether CFMs, in particular CFM-4.16, would inhibit parental and drug-resistant NSCLC cells. As a proof-ofconcept study, we treated the A549 and H1299 NSCLC cells with various doses of CFM-4 and its analogs CFM4.6, -4.16 and -4.17 , and determined the viabilities of these cells by MTT assays. A $10 \mu \mathrm{M}$ and $20 \mu \mathrm{M}$ dose of each of the compounds caused significant loss of viability of both the NSCLC cells (Figure 1A). Although treatments with a $5 \mu \mathrm{M}$ dose of CFM-4, -4.16 , or -4.17 also resulted in reduced viabilities of NSCLC cells when compared 
with their respective, DMSO-treated controls, CFM-4.16 was generally more potent when compared with other three compounds (Figure 1A). Further dose response analyses with reference to A549 and H1299 NSCLC cells (Figure 1A) and other NSCLC cells with mutant EGFR (see below) revealed that $\mathrm{GI}_{50}$ and $\mathrm{LC}_{50}$ of CFM-4.16 were $2.0 \mu \mathrm{M}$ and 5-5.8 $\mu \mathrm{M}$ respectively (not shown).

We next determined whether CFMs also inhibit growth of the EGFR TKI-resistant NSCLCs. We first developed and characterized NSCLC cells that were resistant to EGFR TKIs Erlotinib, Rociletinib, or Osimertinib by culturing them in the continual presence of the respective TKIs until resistance was observed. Since, Erlotinib is frequently used in clinic for treatment of the NSCLC tumors with activating mutation in the kinase domain of EGFR [4], we chose the HCC 827 NSCLC cells with EGFR exon $19(\Delta 19)$ mutation for generation of the Erlotinib-resistant cells. As shown in Table 1, the $\mathrm{GI}_{50}$ doses of Erlotinib for parental and resistant HCC 827 cells were $\sim 0.1 \mu \mathrm{M}$ and $\geq 15 \mu \mathrm{M}$, respectively.
With growing evidence suggesting that development of resistance the TKIs Erlotinib or Gefitinib often involves activation as well as overexpression of other RTKs such as cMet or Alk, a significant subset of resistant tumors often also acquire additional, activating mutations in EGFR kinase domain. These mutations include the L858R change as well as the "gatekeeper" T790M substitution that collectively render EGFR to become constitutively active [4]. Additional allosteric, non-ATPcompetitive EGFR TKIs were recently identified and the two compounds Rociletinib and Osimertinib were tested in clinical trials with subsequent and recent FDA approval of Osimertinib for use in treatment of resistant NSCLCs. Since recent laboratory studies have reported development of resistance to Rociletinib or Osimertinib in NSCLC cells [5], we chose H1975 NSCLC cells with EGFR T790M and L858R mutations for generation of Rociletinib or Osimertinib-resistant cells. The $\mathrm{GI}_{50}$ doses for Rociletinib and Osimertinib for the parental H1975 cells were 0.18 and $0.17 \mu \mathrm{M}$, respectively. Although the
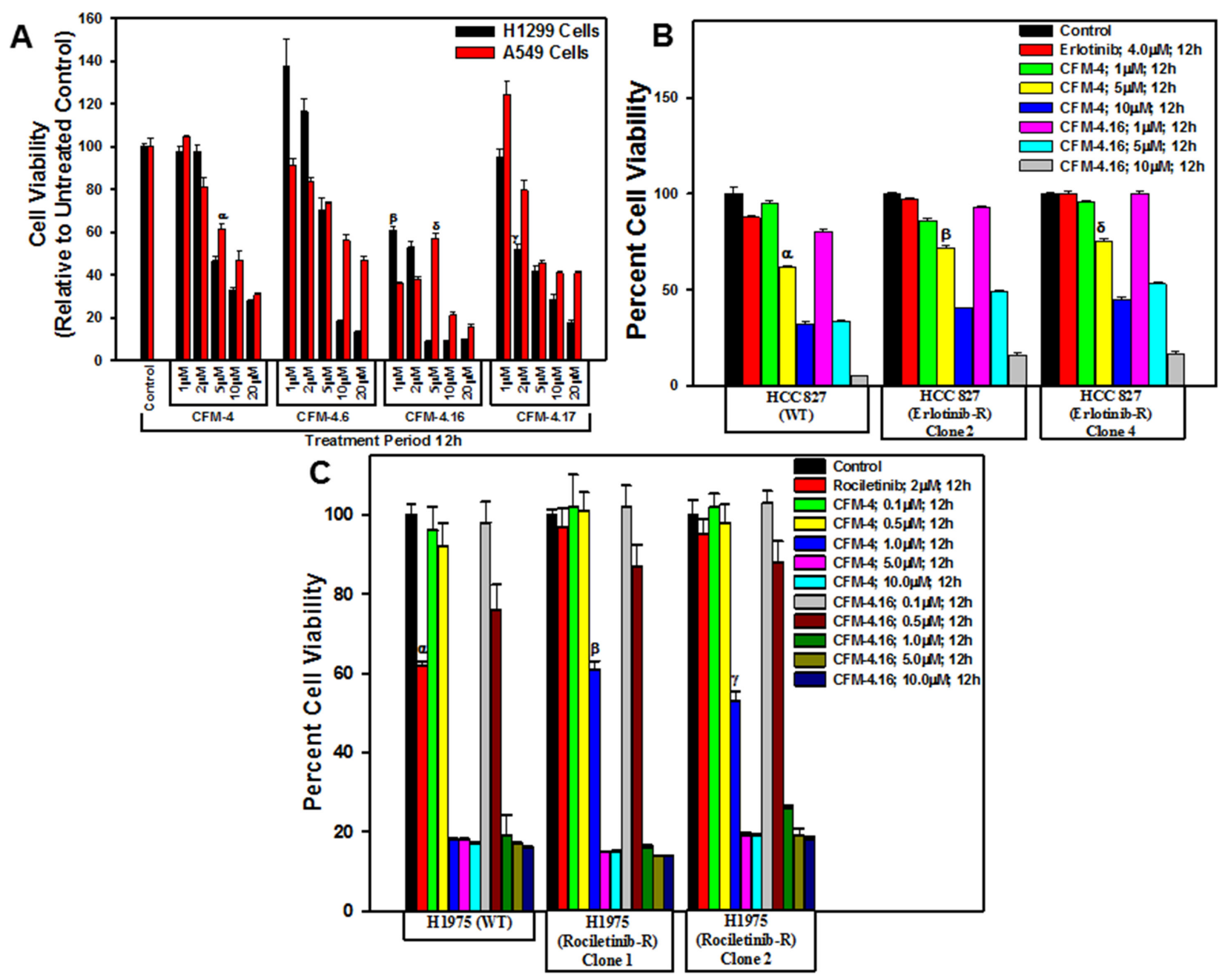

Figure 1: CFMs inhibit NSCLC cell growth. Noted cell lines were either treated with DMSO (Control), with various CFMs (A-C), Erlotinib (B), or Rociletinib (C) for indicated dose and time. Cell viability was determined by MTT assay. The data in the histograms represent means of three independent experiments; bars, S.E. $\alpha, \beta, \gamma, \delta, p \leq 0.05$ relative to the respective DMSO-treated controls. 
Table 1: GI50 values of parental and TKI-resistant NSCLC cells

\begin{tabular}{|c|c|c|}
\hline \multirow{2}{*}{$\begin{array}{l}\text { NSCLC } \\
\text { cell line }\end{array}$} & \multicolumn{2}{|l|}{ Erlotinib (72 h) } \\
\hline & & $\mathrm{GI}_{50}(\mu \mathrm{M})$ \\
\hline \multirow{2}{*}{$\begin{array}{l}\text { HCC } 827 \\
(\text { EGFR } \Delta 19)\end{array}$} & Wild type & $\sim 0.1$ \\
\hline & Erlotinib-R Clones 1-5 & $\geq 15.0$ \\
\hline \multicolumn{3}{|c|}{$\begin{array}{ll}\text { Rociletinib (72 h) } \\
\end{array}$} \\
\hline \multirow{7}{*}{$\begin{array}{l}\text { H1975 } \\
\text { (EGFR T790M plus L858R) }\end{array}$} & & $G I_{50}(\mu M)$ \\
\hline & Wild type & $<0.18$ \\
\hline & Rociletinib-R Clone 1 & $\sim 8.0[\geq 0.5]$ \\
\hline & Rociletinib-R Clone 2 & $\sim 7.5[\geq 0.5]$ \\
\hline & Rociletinib-R Clone 3 & $\sim 6.5$ \\
\hline & Rociletinib-R Clone 4 & $\sim 6.0$ \\
\hline & Rociletinib-R Clone 5 & $\sim 4.5$ \\
\hline \multirow{4}{*}{$\begin{array}{l}\text { H1975 } \\
\text { (EGFR T790M plus L858R) }\end{array}$} & \multicolumn{2}{|c|}{$\begin{aligned} & \text { Osimertinib (72 h) } \\
&\end{aligned}$} \\
\hline & & $\mathrm{GI}_{50}(\mu \mathrm{M})$ \\
\hline & Wild type & 0.17 \\
\hline & Osimertinib-R pools & $\sim 12.0$ \\
\hline
\end{tabular}

The parental and erlotinib-resistant (Erlotinib-R) sublines were treated with 0.1, 0.5, 1.0, 2.0, 4.0, 6.0, 8.0, 10.0, and 15.0 $\mu \mathrm{M}$ dose of Erlotinib for $72 \mathrm{~h}$. In the case Rociletinib-resistant cells, the respective parental and resistant (Rociletinib-R) sublines were treated with $0.1,0.2,0.5,1.0,2.0,5.0$, and $10.0 \mu \mathrm{M}$ dose of Rociletinib for $72 \mathrm{~h}$. In addition, H1975 Rociletinib-R clones 1 and 2 sublines were treated with $0.002,0.005,0.01,0.025,0.05,0.1,0.25$, and $0.5 \mu \mathrm{M}$ Osimertinib for $72 \mathrm{~h}$. Finally, parental and pooled, Osimertinib-resistant $\mathrm{H} 1975$ cells were treated with $0.05,0.1,0.2,0.5,1.0,2.0,5.0$, and $10.0 \mu \mathrm{M}$ Osimertinib. Percent cell viabilities were determined relative to respective DMSO-treated controls. The data in the GI50 columns represent means of three-four independent experiments.

pools of the Osimertinib-resistant H1975 cells had the $\mathrm{GI}_{50}$ dose of $\sim 12 \mu \mathrm{M}$, the $\mathrm{GI}_{50}$ doses of Rociletinib ranged from $\sim 4.5$ to $\sim 8.0 \mu \mathrm{M}$ for the Rociletinib-resistant $\mathrm{H} 1975$ sublines. Of note is the finding that the Rociletinibresistant $\mathrm{H} 1975$ sublines 1 and 2 that elicited $\sim 8.0$ and $7.5 \mu \mathrm{M}$ of Rociletinib $\mathrm{GI}_{50}$ dose respectively, were also resistant to Osimertinib with the $\mathrm{GI}_{50}$ dose of $\geq 0.5 \mu \mathrm{M}$. The data in Table 1 Clearly indicate that all the NSCLC cells developed resistance to the respective TKIs.

To identify the effect of CFM compounds on the growth of the TKI resistant NSCLC cells we performed MTT assays as described in Figure 1A. As shown in Figure $1 \mathrm{~B}$, a dose of $5 \mu \mathrm{M}$ and $10 \mu \mathrm{M}$ of each of CFM-4 or CFM-4.16 inhibited growth of parental and Erlotinibresistant HCC 827 cells. In the case of parental and Rociletinib-resistant H1975 cells, a significant loss of cell viability was also noted when treated with $1 \mu \mathrm{M}$, $5 \mu \mathrm{M}$, or $10 \mu \mathrm{M}$ dose of respective CFMs (Figure 1C). Although both CFMs diminished viability of parental and TKI-resistant NSCLC cells, CFM-4.16 at the doses of 5 $\mu \mathrm{M}$ and $10 \mu \mathrm{M}$ was generally more potent. These data further support our previous findings in TNBC and RCC cells wherein also, we observed CFM-4.16 exhibited increased potency in attenuating cell growth [21, 22]. Taken together, these studies underscore potential of the CFM class of compound(s) to inhibit drug-resistant cancers.

\section{CFM-4.16 suppresses activation of oncogenes in wild-type and TKI-resistant NSCLC cells}

Development of resistance to TKIs erlotinib or gefitinib in NSCLC cells and patient tumors is often associated with abnormal expression and/or activation of oncogenic drivers MET, Alk, Vascular endothelial growth factor receptor (VEGFR), Fibroblast growth factor receptor (FGFR), Src and Abl TKs [23-25]. These TKs regulate development, progression, and metastasis of many cancers including NSCLCs, and often act as drivers of therapeutic resistance in NSCLCs and other cancers [23-25]. We performed immunoblot analyses to investigate molecular pathways involved in NSCLC growth suppression by CFMs, and to identify whether the CFMs targeted oncogenic tyrosine kinases and their signaling. We treated the wild-type and erlotinib-resistant HCC 827 NSCLC cells with erlotinib, CFM-4 or CFM4.16, and the wild-type and rociletinib-resistant H1975 cells with rociletinib and the respective CFM compounds. In addition, we treated the osimertinib-resistant and wildtype H1975 cells separately with osimertinib, CFM-4 or CFM-4.16, and gemcitabine-resistant H23 NSCLC cells with gemcitabine or CFM compounds. Expression levels of Src and MET TKs were analyzed from the lysates through western blotting. As evident from Figure 2 and Supplementary Figure 1A, expression and/or activity of 
MET RTK was observed to be elevated in the TKI-resistant NSCLC cells. Activity and/or expression of Src was high in rociletinib or osimertinib-resistant H1975 cells but not in erlotinib-resistant HCC 827 cells. Treatment with CFM 4.16 reduced the activation and/expression of MET in parental, TKI resistant and gemcitabine resistant NSCLC cells (Figure 2A-2C, and Supplementary Figure 1A). Additionally, treatment with CFM-4.16 resulted in reduced expression and/or activation of Src in parental as well as TKI-resistant H1975 cells but not in parental or erlotinib-resistant HCC 827 cells (Figure 2). These data suggest that CFM-4.16 functions partly by modulating oncogenic kinase signaling pathways to inhibit cell growth. Interestingly, CFM-4.16 not CFM-4, attenuated the activation of STAT3, a transducer of signaling following activation of the EGFR and Src TKs [26, 27], in wildtype or erlotinib-resistant HCC 827 cells (Figure 2A). Furthermore, CFM-4.16 robustly inhibited oncogenic intracellular kinase Akt activity and/or expression in wildtype HCC 827, H1975 and H23 NSCLC cells (Figure 2A, 2B, and Supplementary Figure 1A). CFM-4.16 also inhibited Akt activation in erlotinib and gemcitabineresistant, but not in osimertinib-resistant, NSCLC cells (Figure 2A, 2B, and Supplementary Figure 1A). These data collectively suggest that CFM-4.16 suppresses growth of NSCLC cells in part by reducing the activation/expression as well as downstream signaling of the key survivalregulating oncogenic drivers of drug resistance.

Among the mechanisms of development and maintenance of NSCLC resistance to EGFR TKIs Erlotinib or Gefitinib, amplification and/or activation of MET has been frequently reported in patients as well as in NSCLC cell models of TKI resistance [23, 24]. Accordingly, targeting of MET has been proposed as a potential strategy to overcome resistance to EGFR TKIs and "oncogenic addiction" of the NSCLCs [28]. The NSCLC cell models of resistance to third generation, allosteric inhibitors Rociletinib or Osimertinib that target mutant, constitutively active EGFR tyrosine kinase however have revealed involvement of EGFR-dependent as well as independent mechanisms [5, 29, 30]. Consistent with these observations, our immunoblot studies revealed

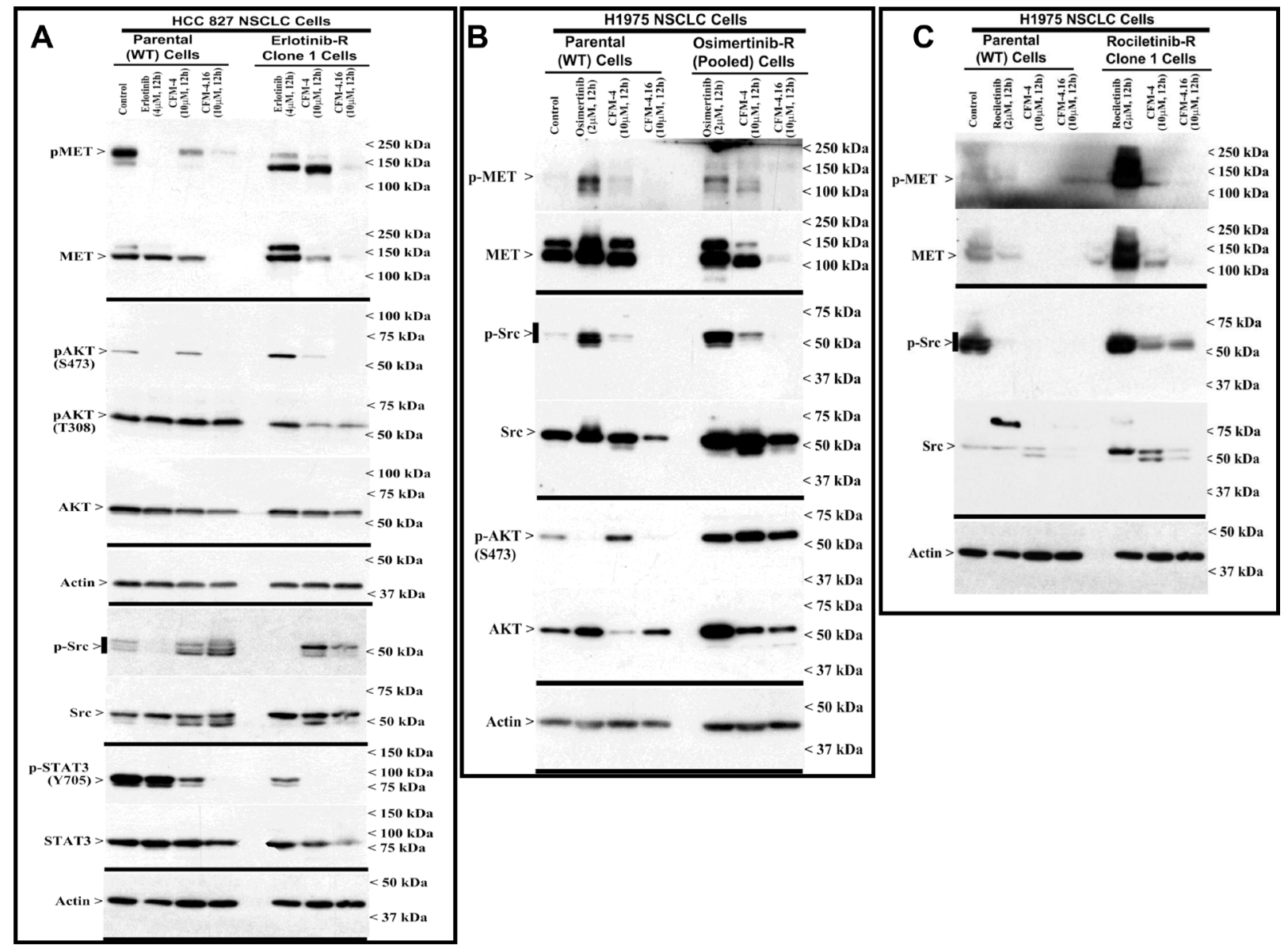

Figure 2: Altered oncogene expression in EGFR TKI-resistant NSCLC cells. (A-C) Indicated parental or TKI-resistant NSCLC cells were either untreated (Control), treated with TKI, CFM-4, or CFM-4.16 for noted dose and time. Cell lysates were analyzed by Western blotting (WB) as in Methods for expression (total) or activation (phosphorylation) of oncogenes MET, AKT, Src, and STAT3. The western blot membranes were subsequently probed with anti-actin antibodies to assess equal loading. The presence of respective protein is indicated by an arrowhead on the left side of each blot. Approximate location of various molecular weight markers is indicated on the right side of each blot. $\mathrm{kDa}$, kilodalton. 
overexpression and activation of MET and Src kinases in erlotinib-resistant HCC 827 cells (see Figure 2A, and Supplementary Figure 2A), while a moderate upregulation of EGFR levels was noted in rociletinib-resistant H1975 cells (Supplementary Figure 2B). We next tested whether CFM-4.16 could have a superior effect in suppressing the growth of the drug resistant cells when used in combination with other well known MET and Src inhibitors. For this study we used FDA-approved Dasatinib, a multi-targeted orally administered inhibitor of RTKs and Src [31] and Tivatinib, an investigational orally administered, highly selective inhibitor of the MET RTK [32]. As shown in Supplementary Figure 2B, treatment of parental or erlotinib-resistant HCC 827 cells with Dasatinib or Tivatinib, following pre-treatment with CFM-4.16, resulted in significantly reduced viabilities when compared with the cells that were treated with each compound separately. Interestingly, as also shown in Supplementary Figure 2D, pre-treatments of wild-type or rociletinib-resistant H1975 cells with CFM-4.16 followed by addition of Gefitinib (EGFR TKI) or Rociletinib also resulted in significantly reduced viabilities when compared with the cells treated with each compound separately. These proof-of-principle findings suggest that CFM-4.16 can sensitize TKI-resistant NSCLC cells to inhibition by TKIs that target EGFR or other oncogenic driver tyrosine kinases.

\section{CFM-4.16 promotes apoptosis in parental and drug-resistant NSCLC cells through activation of c-Jun N-terminal kinase (JNK), stress-activated protein kinases $\mathrm{p} 38$, and enhancing expression of CCAR-1/CARP-1}

We have previously reported that the apoptosis signaling by the chemotherapeutic drugs, Doxorubicin, Gefitinib and Etoposide is mediated through CARP-1

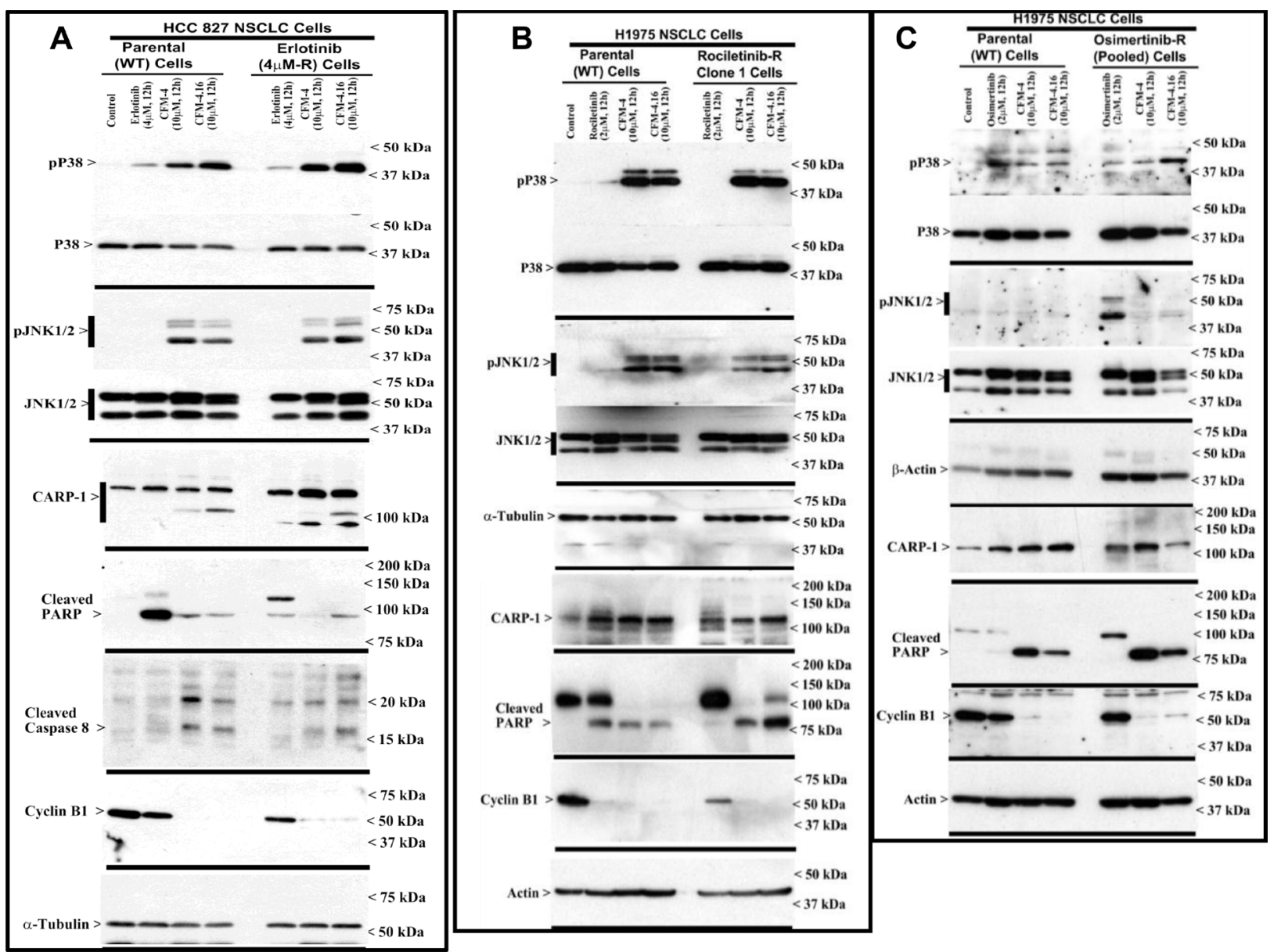

Figure 3: CFM-4.16 stimulates apoptosis in parental and TKI-resistant NSCLC cells in part by upregulating pro-apoptotic CARP-1 and activating SAPKs. (A-C) Indicated parental and TKI-resistant NSCLC cells were either untreated (Control), treated with TKI, CFM-4, or CFM-4.16 for noted dose and time. Cell lysates were analyzed by Western blotting (WB) as in Methods for levels of CARP-1, cyclin B1, cleaved PARP and caspase-8, and activation (phosphorylation) of pro-apoptotic p38 and JNK1/2 SAPKs. The western blot membranes were subsequently probed with anti-actin or $\alpha$-tubulin antibodies to assess equal loading. The presence of respective protein is indicated by an arrowhead on the left side of each blot. Approximate location of various molecular weight markers is indicated on the right side of each blot. $\mathrm{kDa}$, kilodalton. 
[7, 8]. Expression of CARP1 was required for mediating the apoptotic/inhibitory signaling induced by these drugs and also our experimental CFM analogs in TNBC and RCC cells $[21,22]$. Since CFM-4.16 robustly inhibited the growth of wild-type and TKI-resistant NSCLC cells (Figure 1), we further investigated whether expression of CARP1 was necessary for CFM-induced growth inhibition and the molecular mechanisms involved. We observed that equimolar $(10 \mu \mathrm{M})$ dose of CFM-4 or CFM-4.16 induced CARP-1 expression and activation of pro-apoptotic, stressactivated protein kinases (SAPKs) in the wild-type and drug (TKI or gemcitabine)-resistant NSCLC cells (Figure 3, and Supplementary Figure 1B). In addition to activation of SAPK, treatments with CFMs also stimulated cleavage of PARP, activation of caspase 8 , and reduction in levels of mitotic cyclin B1 in the parental and resistant NSCLC cells (Figure 3, and Supplementary Figure 1B). These data suggest that CFMs suppress NSCLC cell growth in part by inducing apoptosis.

To further elucidate TKI resistance molecular pathways downstream of the RTKs, we conducted additional immunoblot analyses utilizing the parental and TKI-resistant NSCLC cells listed in Table 1. These analyses revealed activation of mitogen-activated protein kinase $\mathrm{p} 38 \alpha / \beta$, and NF- $\mathrm{kB}$ subunit p65/RelA in the NSCLC cells that are resistant to Erlotinib or Rociletinib (Supplementary Figure 3A). Activation of NF-kB subunit p65/RelA would be consistent with well-documented roles of proliferation and survival-promoting NF- $\mathrm{KB}$ signaling that is often activated down-stream of a number of activated driver RTKs. The intriguing activation of p38 $\alpha / \beta$ SAPK however in the untreated drug-resistant NSCLC cells as well as in the parental or TKI-resistant NSCLC cells that were treated with CFMs (see Figure 3 and Supplementary Figures 1 and 3) would suggest for known biphasic growth and stress signaling roles, respectively, of $\mathrm{p} 38 \alpha / \beta$ kinase. Environmental stresses and inflammatory mediators activate the core MAPKs that consist of ERK $1 / 2$, JNK $1 / 2 / 3$, and $\mathrm{p} 38 \alpha / \beta / \gamma / \delta$ kinases [33]. MAPKs orchestrate the recruitment of gene transcription, protein biosynthesis, cell cycle control, apoptosis, and differentiation processes. The $\mathrm{p} 38$ and JNKs also regulate stress-dependent inhibition of cellular growth and thus are also known as stress-activated protein kinases (SAPK). A number of pharmacologic inhibitors $p 38 \alpha / \beta$ and JNKs have been discovered and characterized, and tested for use in pre-clinical and clinical settings with respect to cancer [34] and other autoimmune disorders, particularly rheumatoid arthritis [35]. Expectedly, treatments with pharmacological inhibitors of $\mathrm{p} 38 \alpha / \beta$ (Doramapimod/ BIRB796 or Losamapimod), resulted in attenuation/ blockage of p38 activation in TKI-resistant NSCLC cells in a dose-dependent manner, while pre-treatment with Doramapimod or Losamapimod re-sensitized TKIresistant NSCLC cells to respective TKIs (Supplementary Figure 3B, 3C). These proof-of-concept studies underscore a significant and novel mechanism of TKI resistance, and reveal presence of signaling "node" with potential to permit development of additional targeting strategies for treatment and management of RTK-driven resistant NSCLCs.

To investigate whether CARP-1 expression is necessary for the CFM mediated cell growth inhibition of the NSCLC cells, we generated stable sublines of HCC 827 expressing plasmid encoding CARP-1 antisense or its vector as described previously [7]. The stable cell lines generated were then characterized, by measuring the levels of CARP-1 as shown in Figure 4A. In the cells expressing the anti-sense plasmid, CARP-1 expression was reduced compared to the cells expressing the vector and the parental cells. The characterized clones were then treated with CFM-4.16 and TKI Erlotinib and the viabilities of the cells were measured. We observed that in the HCC 827 cells expressing CARP-1 antisense, the inhibitory effects of CFM-4.16 and Erlotinib was significantly reduced compared to the vector plasmid expressing cells (Figure 4B). These findings suggest that the CFMs inhibit growth of parental as well as drug resistant NSCLC cells, and the expression of CARP-1 is necessary to mediate this effect.

\section{CFM-4.16 functions synergistically with B-RAF targeting to inhibit growth of various cancer cells}

In light of our prior studies demonstrating superior inhibition of in vitro and in vivo growth of TNBC cells by a combination of Adriamycin and CFM-4.16 [21], we next investigated whether CFM-4.16 would enhance effectiveness of other, targeted or chemo-therapeutics that are currently used in clinic. To test this hypothesis, we chose compounds that target cell cycle (CDK4/6 inhibitor Palbociclib), DNA repair pathway (PARP inhibitor Olaparib), cell metabolic signaling (mToR1 inhibitor Everolimus), and oncogenic cell growth and survival signaling (B-Raf inhibitors Sorafenib and Dabrafenib). The U.S. Food and Drug Administration (USFDA) recently granted regular approval to Palbociclib (IBRANCE ${ }^{\circledR}$, Pfizer Inc.) for the treatment of hormone receptor (HR) positive, human epidermal growth factor receptor 2 (HER2) negative advanced or metastatic breast cancer in combination with an aromatase inhibitor as initial endocrine based therapy in postmenopausal women [36]. USFDA previously approved Olaparib as monotherapy in ovarian cancer patients with germline BRCA1 mutation [37], and recently expanded Olaparib as maintenance treatment for patients with recurrent epithelial ovarian, fallopian tube, or primary peritoneal cancer who are having partial or complete responses to platinum-based chemotherapy. While Sorafenib serves as one of the frontline therapy for treatment of thyroid, liver, and kidney cancers, Everolimus is a frontline therapy to treat select pancreatic, breast and brain as well as renal 
cancers including a subset of renal cancers where prior treatments with Sorafenib or Sunitinib had failed. Last, but not least, although Dabrafenib was earlier approved by USFDA for treatment of B-Raf V600E mutation-positive un-resectable or metastatic melanoma, recently it was also approved for treatment of B-Raf V600E mutation-positive NSCLC in combination with MEK-targeting therapeutic Tramitinib [38].

Our MTT-based cell viability analyses revealed that each of the targeted therapeutic or CFM-4.16 inhibited viabilities of TNBC, NSCLC, and RCC cells in vitro following treatments with respective compounds. Further analyses of data revealed that cells treated with CFM-4.16 in combination with Olaparib or Palbociclib were additive in their efficacy with combination index $(\mathrm{CI})$ values $\geq 0.8$ (Table 2). The combinatorial treatment of Everolimus and CFM 4.16 however, elicited an additive CI for the NSCLC and RCC cells, their CI values however were $<0.8$ for the TNBC cells suggesting a possible synergy in their mechanisms of action (Table 2). Interestingly, CFM-4.16 in combination with Sorafenib or Dabrafenib consistently elicited CI values that were $<0.8$ for the TNBC, NSCLC and RCC cells with the exception of H1299 NSCLC and UOK262 RCC cells where CI values for CFM-4.16 and Sorafenib but not Dabrafenib combination were $\sim 0.8$ (Table 2). Moreover, with reference to the NSCLC model, although treatments with Sorafenib or CFM4.16 increased expression of CARP-1in parental and Erlotinib-resistant HCC827 cells, CFM-4.16 but not Sorafenib stimulated CARP-1 expression in the parental and Rociletinib- or Osimertinib-resistant H1975 cells (Figure 5A, 5B). However, the combinatorial treatment of Sorafenib and CFM-4.16 failed to induce further CARP-1 increase in these cells. Of note is that although Sorafenib or CFM-4.16 also stimulated a moderate PARP cleavage and loss of cyclin B1 in the parental and their respective, TKI-resistant counterparts, the combined presence of Sorafenib and CFM-4.16 elicited a rather robust cleavage of PARP and RIPK1 proteins as well as loss of cyclin B1 in the parental and TKI-resistant cells (Figures 5A, 5B, $6 \mathrm{~A})$. A combination of both the compounds also induced a robust decline in activities of oncogenes Akt and B-Raf in parental as well as TKI-resistant cells (Figure 6A). Interestingly, our co-immunoprecipitation experiments revealed an interaction between the B-Raf and CARP-1 proteins (Figure 6B, 6C; Supplementary Figure 4). Thus, although CARP-1 is part of the B-Raf proteome, it is likely that CFM-4.16 binding with CARP-1 leads to CARP-1 increase and resultant stress while Sorafenib targeting of B-Raf further inhibits oncogenic/survival signaling and together a combination of both the compounds likely cause elevated levels of stress with consequent robust cell growth inhibitory effects. We have previously found CARP-1 is a co-activator of the anaphase-promoting complex cyclosome (APC/C) E3 ubiquitin ligase (9). Whether and to the extent CARP-1 interaction with B-Raf is involved in ubiquitin-dependent altered expression of $\mathrm{B}-\mathrm{Raf}$ remain to be clarified. Altogether, our findings in Table 2 and Figures 5 and 6 suggest that CFM-4.16 could be a novel, sensitizer for Raf targeting therapeutics in various types of cancers.

\section{CFM-4.16 inhibits migration and three- dimensional growth of the parental and TKI- resistant NSCLC cells}

Since CFM-4.16 robustly disrupted integrity of the tubules formed by human umbilical vein endothelial cells,
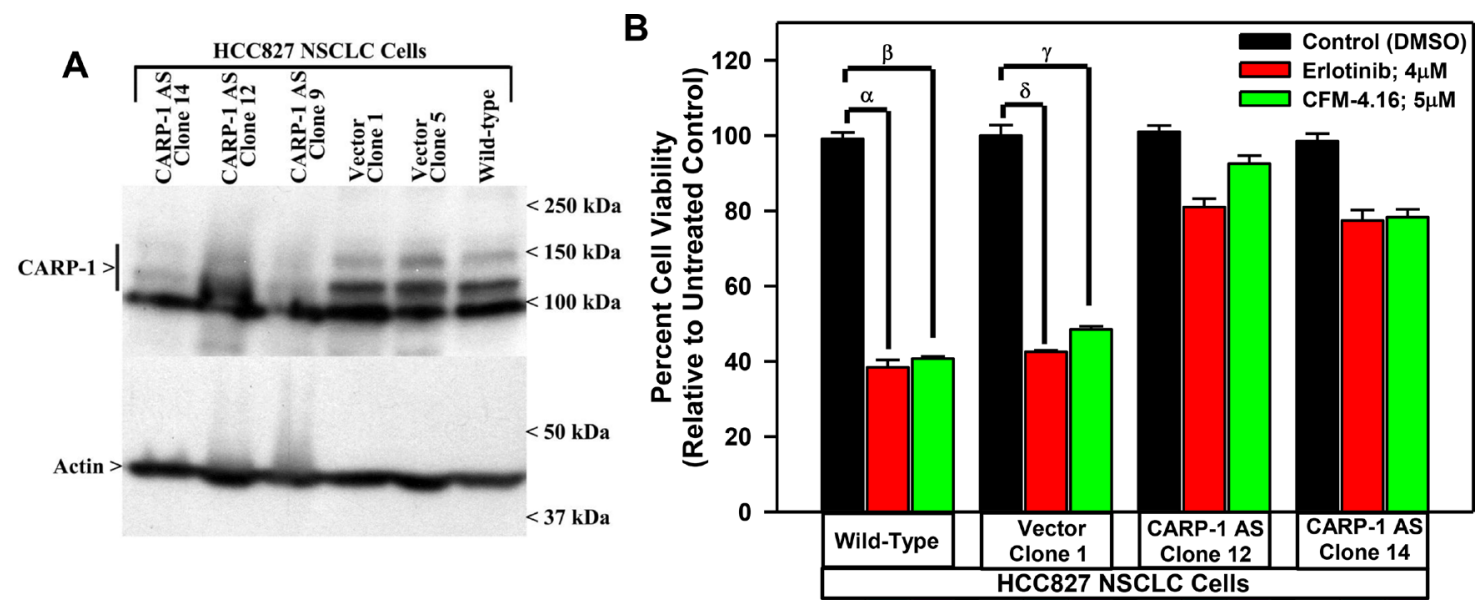

Figure 4: Knockdown of CARP-1 blocks CFM-4.16 effects. (A) Cells were either untransfected, transfected with the pcDNA3/ Hygro vector plasmid or plasmid expressing CARP-1 antisense, and stable, hygromycin-resistant cells were generated and characterized as detailed in methods. Cell lysates from indicated stable cell lines were subjected to WB analysis as in Figure 3 for levels of CARP-1 and actin. (B) The Indicated vector or CARP-1 antisense expressing NSCLC sublines were treated with DMSO (Control), noted doses of CFM4.16, or Erlotinib for $24 \mathrm{~h}$. Cell viability was determined by MTT assay. The histogram columns represent means of three independent experiments; bars, S.E. $\alpha, \beta, \gamma, \delta, p \leq 0.05$ for the Erlotinib or CFM-4.16-treated wild-type or the vector expressing subline relative to their respective DMSO-treated control. 
Table 2: Combination index (CI) values of CFM-4.16 plus various targeted therapeutics

\begin{tabular}{|c|c|c|c|c|c|c|}
\hline \multirow[b]{2}{*}{ Cell Lines } & \multicolumn{6}{|c|}{ Combination Index Drug + CFM-4.16 } \\
\hline & Sorafenib & Everolimus & Olaparib & Palbociclib & Ulixertinib & Dabrafenib \\
\hline MDA-MB-231 (TNBC) & 0.256 & 0.317 & 1.077 & 0.557 & 0.393 & 0.4231 \\
\hline HCC-1937 (TNBC) & 0.84910 & 0.44371 & ND & ND & 0.715 & 0.7012 \\
\hline Hs-578T (TNBC) & 0.6512 & 0.5121 & ND & ND & 0.733 & 0.4653 \\
\hline MDA-MB-468 (TNBC) & 0.689 & 0.699 & 1.305 & 0.811 & 0.655 & 0.498 \\
\hline HCC-70 (TNBC) & 0.68 & 0.698 & 1.305 & 0.811 & ND & 0.626 \\
\hline HCC-1806 (TNBC) & 0.69614 & 0.27961 & ND & ND & 0.554 & 0.4121 \\
\hline H460 (NSCLC) & 0.147 & 0.313 & 1.194 & 0.818 & ND & 0.512 \\
\hline H1299 (NSCLC) & 0.84 & 0.79623 & 1.59868 & 1.07999 & ND & 0.612 \\
\hline HCC-827 (NSCLC) & 0.35800 & 0.81829 & 1.1987 & 0.9213 & ND & 0.15672 \\
\hline H-1975 (NSCLC) & 0.47810 & 0.54892 & 1.1 & 0.9542 & ND & 0.21412 \\
\hline A498-wild type (RCC) & 0.53 & 0.7 & 1.0978 & 0.7 & ND & 0.13850 \\
\hline $\begin{array}{l}\text { A498-Everolimus } \\
\text { Resistant type (RCC) }\end{array}$ & 0.6 & 1.3 & 1.121 & 0.971 & ND & 0.677 \\
\hline UOK262-wild type (RCC) & 0.76 & 0.67 & 0.9878 & 0.897 & ND & 0.234 \\
\hline $\begin{array}{l}\text { UOK262-Everolimus } \\
\text { Resistant type (RCC) }\end{array}$ & 0.8 & 1.45 & 1.312 & 1.0023 & ND & 0.587 \\
\hline MDA-MB-231 (TNBC) & 0.53 & 0.313 & 1.194 & 0.818 & ND & 0.512 \\
\hline
\end{tabular}

Abbreviations: Sorafenib, Dabrafenib: B-Raf Inhibitors. Everolimus: mToR Inhibitor. Olaparib: PARP Inhibitor. Palbocicilib: CDK4/6 Inhibitor. Ulixertinib: ERK1/2 Inhibitor. ND: Not Done; TNBC: Triple-negative Breast Cancer; NSCLC: Non-small Cell Lung Cancer; RCC: Renal Cell Carcinoma.

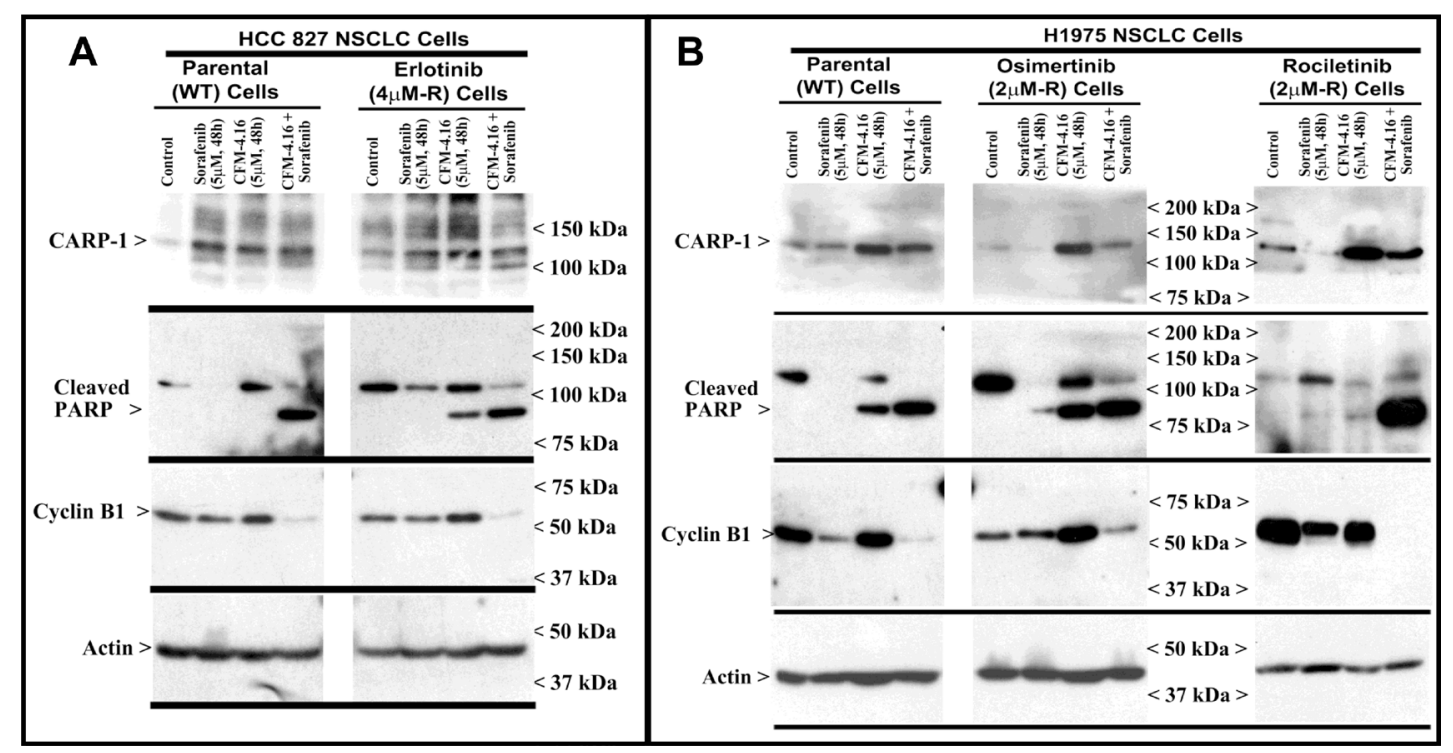

Figure 5: Apoptosis induction in parental and TKI-resistant NSCLC cells following treatments with CFM-4.16 and Sorafenib. (A, B) Indicated parental and TKI-resistant NSCLC cells were either untreated (Control), treated with Sorafenib, CFM-4.16, or a combination of Sorafenib and CFM-4.16 for noted dose and time. Cell lysates were analyzed by Western blotting (WB) as in Methods for levels of CARP-1, cleaved PARP, cleaved RIPK1, activated and total Akt and B-Raf kinases, and expression of cyclin B1 as in Figure 3. The western blot membranes were subsequently probed with anti-actin antibodies to assess equal loading. The presence of respective protein is indicated by an arrowhead on the left side of each blot. Approximate location of various molecular weight markers is indicated on the right side of each blot. kDa, kilodalton 
suppressed TNBC cell growth and migration as colonies in soft agar and 3-dimensional cultures in vitro, we tested whether CFM-4 or -4.16 compounds will also influence the biological properties of migration and 3-dimensional growth of Erlotinib- or Gemcitabine-resistant NSCLC cells. We observed that treatment with CFM-4 or CFM4.16 prevented the growth of parental and Erlotinib or Gemcitabine-resistant NSCLC cells into the areas of wound caused by a scratch (Supplementary Figure 5A-5E). We also noted significant attrition in size and number of colonies formed by the parental and Erlotinib or Gemcitabine-resistant NSCLC cells in soft agar upon treatment with CFM-4 or CFM-4.16 (Supplementary Figure 6A-6C). We have previously observed significant reduction in the growth of mammospheres and shperoids derived from TNBC and RCC cells, respectively, upon treatments with CFM-4.16 [21, 22] We investigated whether a similar inhibitory effect occurs on spheroids derived from NSCLC cells when treated with CFM-4.16. As depicted in Figure 7, the parental HCC 827, H1975 cells and also their respective, TKI-resistant sublines formed NSCLC spheroids. Similar to our previous observations in TNBC and RCC models, treatment with CFM-4.16 resulted in disintegration of spheres of both

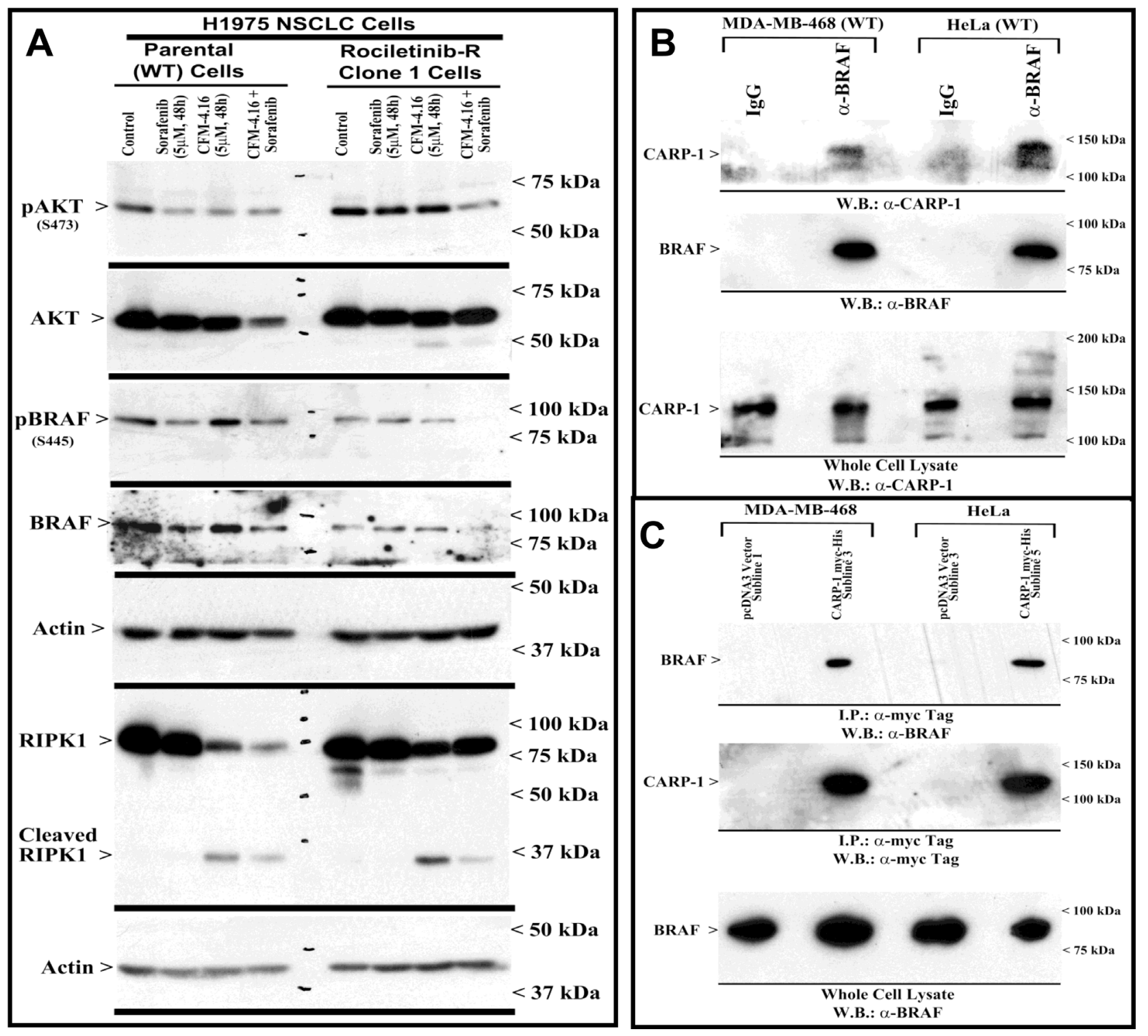

Figure 6: CFM-4.16 and Sorafenib combination inhibits B-Raf activity, and CARP-1 interacts with B-Raf. (A) Indicated parental and TKI-resistant NSCLC cells were either untreated (Control), treated with Sorafenib, CFM-4.16, or a combination of Sorafenib and CFM-4.16 for noted dose and time. Levels of actin, total and cleaved RIPK1, activated and total Akt and B-Raf kinases were analyzed by western immunoblotting essentially as in Figure 5. (B, D) Approximately $1 \mathrm{mg}$ of cell lysate from each of the indicated cells was subjected to immunoprecipitation using anti-B-Raf (B) or anti-myc-tag (C) antibodies. The immunoprecipitates were then analyzed by WB. In B, the membrane was probed with anti-CARP-1 ( $\alpha 2)$ antibodies (7) or B-Raf antibodies while the membrane in C was probed with $\mathrm{B}-$ Raf or myc-tag antibodies. The presence of respective protein is indicated by an arrowhead on the left side of each blot. Approximate location of various molecular weight markers is indicated on the right side of each blot. kDa, kilodalton. 
the parent as well as TKI resistant human NSCLC cells (Figure 7).

\section{Combinatorial treatment of CFM-4.16 NLF with Sorafenib causes superior inhibition of xenografted, TKI rociletinib-resistant NSCLC tumors}

We have previously developed and tested nanolipid formations (NLFs) of CFM-4 and CFM-4.16 to address the issues of poor bioavailabity and poor aqueous solubility [20-22]. These NLFs resulted in significant improvements in overall bioavailabilities of CFM-4 and CFM-4.16 [20, 21]. On the basis of our current findings in Table 2 that suggested a synergistic NSCLC inhibitory mechanism of CFM-4.16 when used in combination with B-Raf targeting, we investigated the anti-tumor efficacy of CFM-4.16 NLF in combination with Sorafenib, in-vivo. Nude mice bearing Rociletinib-resistant NSCLC H1975 orthotropic xenograft tumors were treated with CFM-4.16 NLF, Sorafenib, or a combination of both by oral gavage as described in methods and our published protocols [20,21]. As depicted in Figure 8A, the control (placebo) and CFM4.16 NLF groups did not show significant tumor growth inhibition. Although oral administration of Sorafenib showed reduction in tumor volume over a 13 and 19-day period, the combinatorial treatment of CFM-4.16 NLF and Sorafenib resulted in significantly reduced tumor volume when compared with treatments of compounds on their own. Our result showed that from all the formulations evaluated, combination of CFM-4.16 NLF and Sorafenib was able to significantly reduce tumor burden after 13 $(p<0.05)$ and $19(p<0.01$ and $p<0.001)$ days when compared with the control group and group of animals treated with CFM-4.16 NLF alone. The other formulations could not decrease the tumor burden significantly when compared with the control. We then performed immunohistological analysis of a representative NSCLC tumor from the animals treated with placebo (control), CFM4.16 NLF, Sorafenib, or combination of CFM-4.16 NLF and Sorafenib. We observed increased staining for TUNEL and CARP-1 protein in the tumors from the treated animal when compared with the tumor from the control, placebotreated animal (Figure 8B). Of note is fact that consistent with our data in Figure 5B where a higher levels of CARP1 were noted in the Rociletinib-resistant H1975 cells, staining for CARP-1 were rather intense in the tumor derived from the animal treated with CFM-4.16 NLF. The tumor from the animal treated with a combination of CFM-4.16 NLF and Sorafenib had robust CARP-1 levels when compared with the tumor derived from the animal treated with Sorafenib only. The data in Figure 8 collectively demonstrate that CFM-4.16 enhances efficacy of Sorafenib to inhibit TKI-resistant NSCLC tumor in vivo. These findings would be consistent with our current in vitro observations as well as our previous studies where
CFM-4.16 stimulated apoptosis in a variety of cancer cell types including those of TNBC and RCC origins [21, 22].

\section{DISCUSSION}

We had previously identified CARP-1/CCAR1, a perinuclear phosphoprotein, which is a biphasic regulator of chemotherapy-induced apoptosis as well as steroid-induced activation of steroid/thyroid nuclear receptors $[7,8,11,12]$. Our recent chemical biology studies culminated in identification of small molecule compounds termed CARP-1 functional mimetics (CFMs) that bind with CARP-1 and stimulate apoptosis in various cancer cells [9, 17-22]. CFM-4 analog -4.16 elicited superior inhibition of only the TNBC cells in vitro and TNBC cell-derived xenografted tumors in vivo when administered in combination with the chemotherapeutic Adriamycin [21]. CFM-4.16 was also superior in inhibiting growth of parental and mammalian target of rapamycin (mToR) inhibitor Everolimus-resistant renal cancer cells (RCC) in vitro and in vivo [22]. Although, CFM compounds including CFM-4.16 are not watersoluble and consequently have poor bioavailability, nanolipid formaulations significantly enhanced bioavailabilities of these compounds following their oral or intravenous administration when compared with similarly administered free compounds in rodents [20-21]. Given that CARP1 is also a transducer of inhibitory signaling following targeting of EGFR [8], and the fact that previous studies also demonstrated CARP-1 antagonism of EGFR signaling in C. elegans [16], prompted us to investigate whether we could exploit CARP-1 signaling for inhibition of cancers that develop resistance to EGFR-targeted therapeutics. We tested this hypothesis in the current study in conjunction with a subset of NSCLC cells. These established NSCLC cell models are representative of patient tumors that often harbor mutant, constitutively active EGFR where treatments with EGFR TKIs are frontline options, and development of resistance to various EGFR-targeting TKIs continues to be a challenge in clinical management of this disease.

A wealth of recent investigations have reported a variety of redundant, EGFR-independent mechanisms of NSCLC resistance to EGFR TKIs in clinic as well as in pre-clinical NSCLC models. Consistent with these crucial mechanistic findings of development of EGFR TKI-resistant NSCLCs, additional therapies targeting resistant NSCLCs have proven beneficial in clinic. Yet the progression-free survival in patients with resistant NSCLC remains unacceptably low. To begin to test potential of CFM compounds in inhibiting TKI-resistant NSCLCs, we first obtained and characterized EGFR mutant NSCLC cells with induced resistance to chronic presence of TKIs. We chose HCC 827 cells with EGRR $\Delta 19$ mutation and exposed these cells to TKI Erlotinib. Additionally, we exposed the H1975 cells with EGFR L858R and 
the 'gatekeeper' T790M mutations to TKIs Rociletinib or Osimertinib. As shown in Table 1, we obtained a number of TKI-resistant NSCLC cells that were further characterized. Consistent with a number of prior reports [4, 5, 28], Erlotinib resistant HCC827 cells had elevated levels and activities of MET and its downstream Src and Akt kinases. Rociletinib-resistant H1975 cells also had elevated expression and activities of MET. Moreover, in agreement with prior findings [29], we found a moderate increase in expression but not activation of EGFR in these Rociletinib-resistant NSCLC cells. Osimertinibresistant H1975 cells, however, had increased expression and activities of Src and Akt kinases. Together with significantly elevated IC50 values that were noted for the respective TKI-resistant sublines that are summarized in Table 1, our studies suggest for emergence of robust, TKI-resistant phenotypes of EGFR-mutant NSCLC cells. Importantly, our data in Supplementary Figure 2, also suggest a proof-of-concept targeting of the parental or the Erlotinib-resistant NSCLC cells by CFM-4.16

3-D Sphere Formation Assay (1 Week)
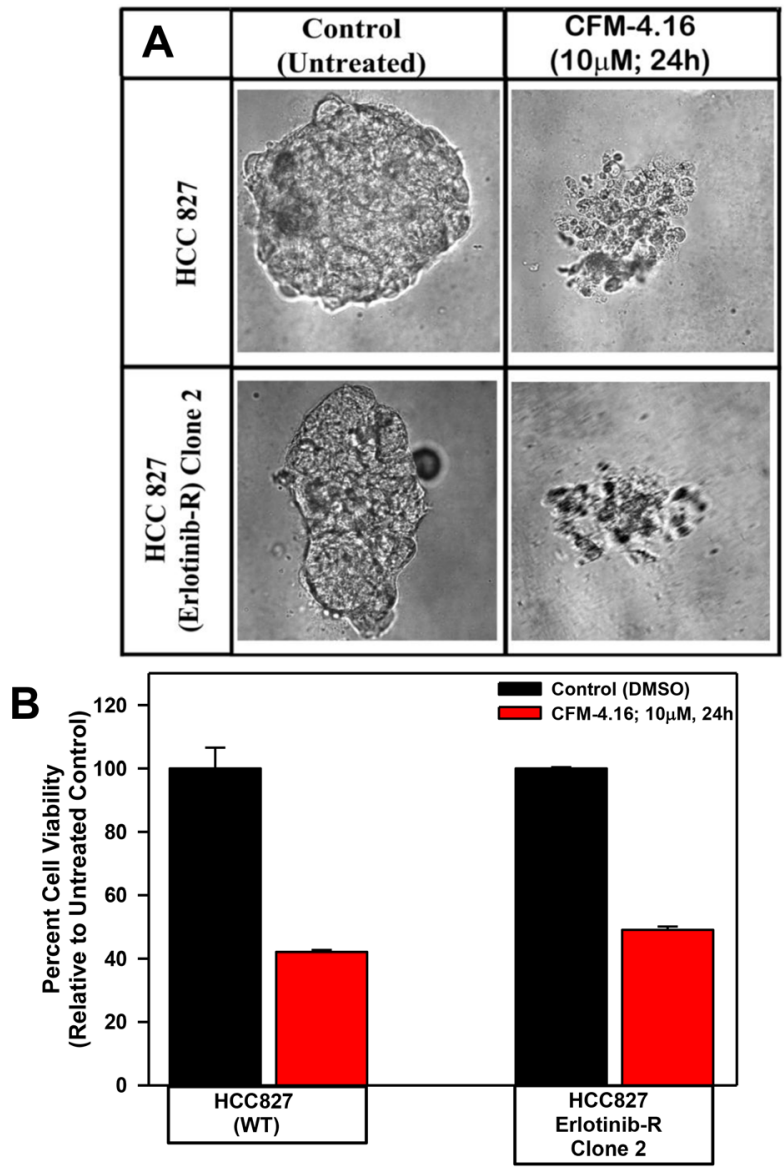

in combination with Src targeting (Dasatinib) or MET targeting (Tivatinib). Similarly, although CFM-4.16 in combination with Rociletinib elicited higher efficacy in inhibiting parental or rociletinib-resistant H1975 cells, CFM-4.16 in combination with EGFR TKI Gefitinib caused greater inhibition of Rociletinib-resistant H1975 cells when compared with their parental counterparts. These preliminary studies suggest that CFM-4.16 compound could potentially enhance effectiveness of current TKIs in inhibiting parental and, more importantly, EGFR TKI-resistant, NSCLC cells.

A novel aspect of our current studies is the finding that the compound CFM-4.16 functions synergistically with therapeutics that target Raf kinases (Sorafenib or Dabrafenib; Table 2). Interestingly, we noted synergism between CFM-4.16 and Sorafenib or Dabrafenib for a number of NSCLC, TNBC as well as RCC cells. Sorafenib is a multikinase inhibitor that targets RTKs such as VEGFR and PDGFR, as well as the C- and B-Raf kinases [39, 40]. The fact that Dabrafenib, a B-Raf kinase inhibitor that

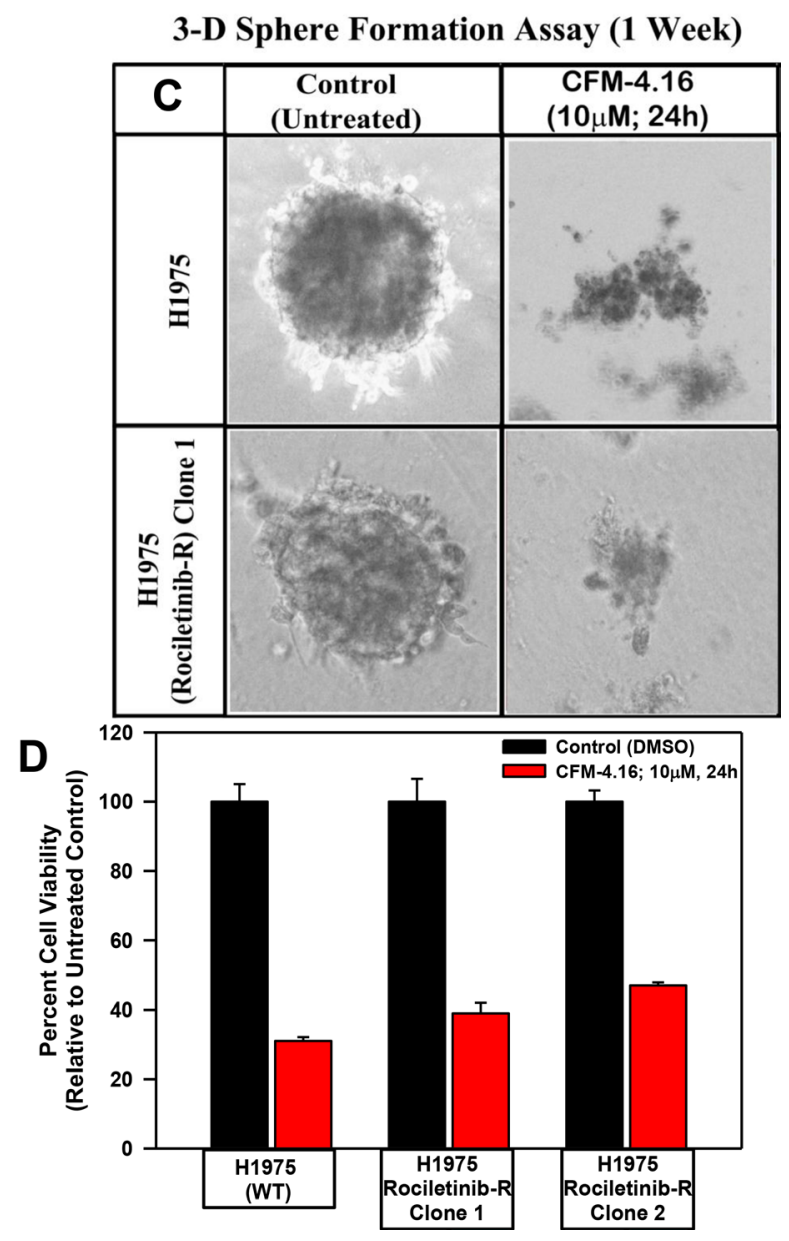

Figure 7: CFM-4.16 inhibits growth of NSCLC spheres derived from parental and TKI-resistant cells. Parental and TKI-resistant NSCLC cells were grown as spheres as detailed in Methods. The sphere cultures were either untreated (Control) or treated with CFM4.16 for noted dose and time. The untreated and treated spheres were then photographed (A, C) or the cells were subjected to MTT-based viability assay as in Figure 1 (B, D). Representative photomicrographs of untreated and CFM-4.16 treated spheres are shown in panels A and C. The histograms in panels B and C represent means of three independent experiments, bars, S.E. 
is used as single agent treatment for patients with B-Raf V600E mutation-positive advanced melanoma as well as in combination with MEK inhibitor Trametinib for advanced or metastatic NSCLCs [38, 41, 42], also synergized with CFM-4.16 would strongly argue for Raf targeting being synergistic with CFM-4.16 in a variety of cancer cells. Since CFM-4 functions in part by binding with CARP-1 and stimulating CARP-1-mediated stress and apoptotic signaling [9, 10, 20-22], the synergistic actions of CFM4.16 and Sorafenib then are likely to activate CARP-1dependent stress and apoptosis together with Raf targeting that will impede proliferation and survival. Our findings in Figure 5 further highlight this synergistic aspect of CFM4.16 and Sorafenib functions. Here CFM-4.16 or Sorafenib treatments cause a moderate increase in PARP cleavage as well as decline in levels of mitotic cyclin B1 in both the parental and EGFR TKI-resistant NSCLC cells. However, we noted a robust cleavage of PARP and RIPK1 proteins, diminished activities of oncogenic Akt and B-Raf kinases, as well as cyclin B1 loss in CFM-4.16 plus Sorafenibtreated cells.

Last, but not least, we tested the potential of CFM4.16 in combination with Sorafenib to inhibit growth of xenografted, Rociletinib-resistant H1975 NSCLC cellderived orthotopic tumors in immunocompromised (nude) mice. Here, we administered Sorafenib, CFM-4.16 NLF, or a combination of both the agents by oral gavage on the alternate days over a two-week period. The combination of CFM-4.16 and Sorafenib provoked a significantly reduced tumor volumes when compared with those noted in the
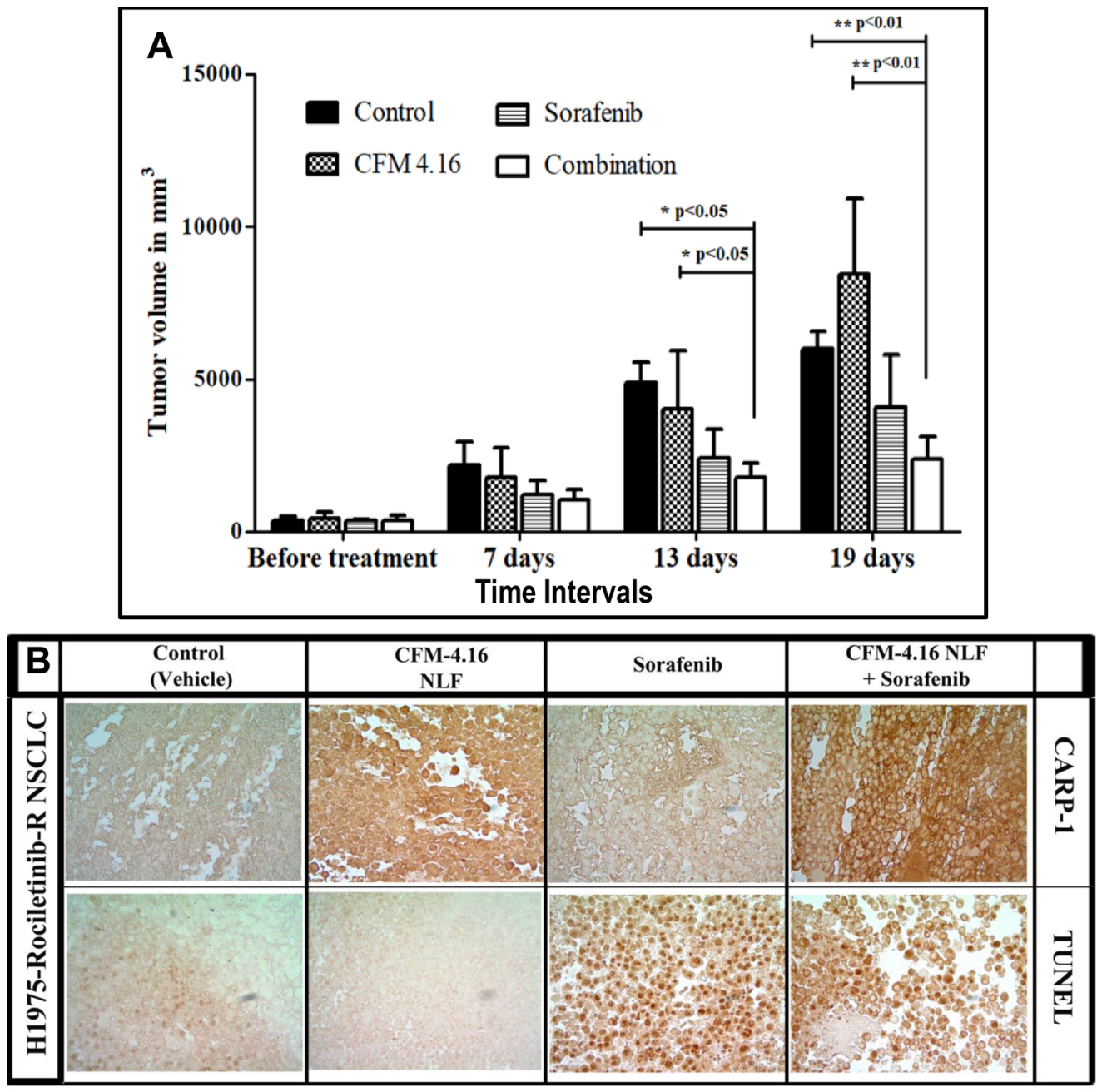

Figure 8: Nano-lipid formulation of CFM-4.16 in combination with Sorafenib inhibits growth of TKI-resistant NSCLC cellderived xenografts. (A) Histogram showing tumor volume of the vehicle-treated (indicated as Control), CFM-4.16-NLF, Sorafenib, or CFM-4.16 plus Sorafenib-treated, Rociletinib-resistant NSCLC (H1975) xenograft-bearing animals. The xenograft establishment, treatment and analysis procedures were carried out essentially as detailed in Methods. The columns represent average values from a total of six animals in respective group, bars, SE, significant where ${ }^{* *} p=0.01$ vs Control. (B) CFM-4.16-NLF and Sorafenib treatments (po) induce CARP-1 expression and apoptosis in NSCLC tumor xenografts. NSCLC tumor xenografts generation and animal treatments were as in Methods. Representative tumor tissues from two animals each from the Control or treated groups were fixed in formalin, paraffin embedded, processed, and subjected to immuno-staining as detailed in Methods. Photomicrographs $(400 \times$ magnification $)$ are shown for apoptosis (by TUNEL assay), and levels of CARP-1 protein as noted in methods. 
control, placebo-treated animals. Together with our in vitro studies demonstrating a robust synergism between CFM-4.16 and Sorafenib, our current data provide us with a further rationale to develop CFM compounds and their formulations for sensitizing parental and resistant NSCLCs to Raf-targeting therapeutics.

\section{MATERIALS AND METHODS}

\section{Cell culture, reagents and chemicals}

Structure and synthesis of CFM-4, -4.16, and -4.17 compounds have been recently described $[21,22]$. A stock solution of 10-50 mM of each CFM was prepared in dimethyl sulfoxide (DMSO) and stored at $-20^{\circ}$ C. 3-[4,5-Dimethylthiazol-2-yl]-2,5diphenyltetrazolium bromide (MTT) were obtained from Sigma-Aldrich, St Louis, MO, USA). The mToR inhibitor Everolimus, PARP inhibitor Olaparib, CDK4/6 inhibitor Palbocicilib, Raf inhibitors Sorafenib and Dabrafenib, Src inhibitor Dasatinib, MET inhibitor Tivatinib, p38 inhibitors Losmapimod and Doramapimod, as well as the EGFR TKIs Gefitinib, Erlotinib, Rociletinib, and Osimertinib were all purchased either from SelleckChem, Boston, MA, USA or ApexBio, Houston, TX, USA. The ERK1/2 inhibitor Ulixertinib was obtained from Chemietek, Indianapolis, IN, USA. Each compound was dissolved in DMSO to obtain a $50 \mathrm{mM}$ stock solution and stored at -20 until needed. We purchased all other analytical reagent grade chemicals from Sigma-Aldrich (St Louis, MO) and used them without further purification.

DMEM, EMEM medium and antibiotics (penicillin and streptomycin) used in this study were purchased from Invitrogen Co. (Carlsbad, CA, USA). Fetal bovine serum (FBS) and DMSO were obtained from Denville Scientific Inc. (Metuchen, NJ, USA), and Fisher Scientific (Fair Lawn, NJ, USA), respectively. The Protein Assay Kit was purchased from Bio-Rad Laboratories (Hercules, CA, USA). The mouse monoclonal antibodies for $b-$ actin were acquired from Sigma-Aldrich (St. Louis, MO, USA). We purchased antibodies for $\alpha$-tubulin (rabbit polyclonal), Cyclin B1 (V152, mouse monoclonal), Cleaved Caspase-8 (IC12, mouse monoclonal), cleaved PARP (Asp214; mouse monoclonal), RIPK1 (Cat\#3493; rabbit monoclonal), phospho (T180/Y182) and total p38 $\alpha / \beta$, phospho (T183/Y185) and total JNK1/2 SAPKs, total and phospho-STAT3 (Y705), total and phosphoMET (Y1234/1235), total and phospho (Y416)-Src, total and phospho-AKT (S473) and T(308), total and phospho (T389)-p70S6K, total and phospho (S2448)-mToR1, total and phospho-(Y1068) EGFR, total and phospho-(S536) p65/RelA NF-кB subunit, total and phospho (S445) B-Raf from Cell Signaling Technology (Beverly, MA, USA). We have previously described generation and characterization of the anti-CARP-1 rabbit polyclonal antibodies [7].

The human NSCLC H1299, A549, H23, H460, $\mathrm{HCC} 827$, and H1975 were obtained from ATCC and validated as described before [43]. The validated TNBC MDA-MB-231, MDA-MB-468, HCC-70, HCC1806, HCC-1937, and Hs578T were obtained from the Karmanos Cancer Institute Biobanking and Correlative Sciences Core and kindly provided by Dr. Julie Boerner. The RCC A498 cells were from ATCC and kindly provided by Dr. Rajvir Dahiya (UCSF). The HLRCC (UOK 262) cells were kindly provided by Drs. Marsten Lanahan (NCI). All the cells were routinely maintained as described before $[38,39]$. All the cell culture media were supplemented with $10 \%$ FBS, 100 units/ml of penicillin, and $100 \mu \mathrm{g} / \mathrm{ml}$ of streptomycin, and the cells were kept at $37^{\circ} \mathrm{C}$ and $5 \% \mathrm{CO} 2$. For cell growth and MTT studies, the cells were cultured in fresh media with $5 \% \mathrm{FBS}$ prior to their treatments with various agents.

\section{Generation of cells resistant everolimus and EGFR TKIs}

Isolation and characterization of Gemcitabineresistant H23 NSCLC cells as well as Everolimusresistant RCC cells have been described before [22, 43]. The NSCLC HCC827 cells were cultured in continuous presence of escalating doses of Erlotinib starting with $100 \mathrm{nM}$ to eventual dose of $4 \mu \mathrm{M}$ over a period of 1 year. Multiple, Erlotinib-resistant sublines were isolated and cultured routinely in $2 \mu \mathrm{M}$ Erlotinib. The H1975 NSCLC cells were separately cultured in continuous presence of escalating doses of Rociletinib or Osimertinib starting with $150 \mathrm{nM}$ to eventual dose of $4 \mu \mathrm{M}$ for each inhibitor over a period of 12 or 6 months, respectively. Multiple, Rociletinib-resistant H1975 cells were isolated and cultured routinely in $2 \mu \mathrm{M}$ Rociletinib. In the case of Osimertinibresistant H1975 cells, a pooled population was obtained and cultured routinely in $2 \mu \mathrm{M}$ Osimertinib. The parental and EGFR TKI-resistant NSCLC cells were characterized for their growth inhibitory $\left(\mathrm{GI}_{50}\right)$ dose of respective TKI by the MTT-based viability assays as below.

\section{Cell viability assays}

The cytotoxicity of CFM-4, -4.16, -4.17, TKIs (Erlotinib, Rocilitinib, Osimertinib, Gefitinib, Dasatinib, Tivatinib), and p38 inhibitors (Losmapimod and Doramapimod) was assessed by MTT assay. First, we seeded $5 \times 10^{3}$ cells in the 96-well plate in triplicate, allowed the cells to grow in fresh culture media for another $24 \mathrm{~h}$, and treated them with respective agents for the noted dose and time. Control cells were treated with $0.1 \% \mathrm{DMSO}$ in culture medium. After treatment, $20 \mu \mathrm{L}$ of $1 \mathrm{mg} / \mathrm{ml}$ of MTT was added to each well and cells were incubated for $2-4 \mathrm{~h}$ at $37^{\circ} \mathrm{C}$. MTT was removed, and the resulting formazan products were dissolved by adding $50 \mu \mathrm{l} \mathrm{DMSO} /$ well. The colorimetric analysis was carried out using a multi-label plate reader at $570 \mathrm{~nm}$ (Victor3; PerkinElmer, Wellesley, MA). 


\section{Combined drug effect analysis}

The median-effect principle of Chou and Talalay was the basis for conducting the CFM-4.16 effect analysis in combination with various targeted therapeutics [44]. The combination index (CI) values were calculated for determining the mode of interaction (synergism, antagonism and additive effect) between CFM-4.16 and therapeutics such as Sorafenib (targets B-Raf), Dabrafenib (targets B-Raf), Everolimus (Targets mToR1), Olaparib (targets PARP), or Palbociclib (targets CDK4/6) as described by Chou and Talalay $[45,46]$. We applied CalcuSyn software version 2 (Biosoft) for above drug combination analysis essentially as described previously [47] and following software guidelines. The CI values presented in Table 2 are based on the effective dose (ED)50 of each compound for the indicated cell line as suggested by the CalcuSyn software.

\section{Generation of CARP-1 knock-down NSCLC cells}

The HCC827 NSCLC parental cells were transfected with vector plasmid pcDNA3/hygro or plasmid expressing CARP-1 anti-sense (Clone 1.6, ref 7). Multiple, stable sublines for hygromycin resistance were selected in the presence of $400 \mathrm{mg} / \mathrm{ml}$ hygromycin (\#10687010, InVitrogen Inc) following methods described before [7]. Knock-down of CARP-1 in multiple, CARP-1 antisense-expressing sublines was determined by western blot analysis of the cell lysates as detailed below. We then determined viabilities of the parental, and vector or CARP-1 antisense plasmid-transfected HCC 827 cells in the presence of CFM-4.16 or Erlotinib by above described MTT assays.

\section{Western blot analysis}

For protein expression analysis, we conducted western blot experiments. The NSCLC cells were treated with $0.1 \% \mathrm{DMSO} /$ Vehicle (Control) or indicated dose and time of the noted compound, and were lysed to prepare protein extracts. Cells were harvested and lysed in RIPA buffer $(50 \mathrm{mM}$ Tris-HCI, $\mathrm{pH}$ 8.0, $150 \mathrm{mM}$ sodium chloride, $1.0 \% \mathrm{NP}-40,0.5 \%$ sodium deoxycholate, $0.1 \%$ sodium dodecyl sulfate (SDS), and $0.1 \%$ of protease inhibitor cocktail) for $20 \mathrm{~min}$ at $4^{\circ} \mathrm{C}$. The lysates were then centrifuged at $14,000 \mathrm{rpm}$ at $4^{\circ} \mathrm{C}$ for $15 \mathrm{~min}$ to get rid of debris. We then determined the protein concentrations of whole cell lysates using the Protein Assay Kit. Supernatant proteins, $50 \mu \mathrm{g}$ from each sample, were separated by SDS$10 \%$ polyacrylamide gel electrophoresis (SDS-PAGE) and transferred to polyvinylidene difluoride (PVDF) membrane (Bio-Rad, Hercules, CA, USA) by standard procedures. The membranes were hybridized with primary antibodies followed by incubation with appropriate secondary antibodies. The antibody-bound proteins were visualized by treatment with the chemiluminescence detection reagent (Amersham Biosciences) according to the manufacturer's instructions, followed by exposure to X-ray film (Kodak $\mathrm{X}$-Omat). The same membranes were then re-probed with either the anti- $\beta$ actin or anti- $\alpha$ tubulin antibody, which was used as an internal control for protein loading.

\section{Preparation and characterization of CFM-4.16 NLF}

The procedure to prepare CFM-4.16 NLF was essentially the same as described in earlier communications [21]. Briefly, appropriate amount of CFM-4.16 was blended with Compritol 888ATO, Miglyol 812N, and Geleol, and the mixture was melted at $70^{\circ} \mathrm{C}$ to form a uniform and clear oil phase. To this, aqueous phase consisting of dispersing surfactant Tween 80 and Vitamin E TPGS in double distilled water was added drop wise to the oil phase at $70^{\circ} \mathrm{C}$. The coarse emulsion was then homogenized for 15 min under high pressure using NanoDebee for about 5 cycles. The formulation was then characterized for its particle size and zetapotential and drug release using methods described elsewhere [20, 21].

\section{Cell migration and clonogenic assays}

The NSCLC cells migration in the absence or presence of CFMs was measured by the "scratch/wound healing" assay essentially as described before [20-22]. Briefly, cells were seeded in a 6-well plate $(\sim 10,000$ cells/well), and when attached, a scratch was created in the cell monolayer using sterile pipette tip. The cells were then allowed to continue growing in the absence (Control) or presence of noted dose of each of the agents for indicated time periods. The cells were photographed at the beginning and at regular intervals during the treatment period, and the images from control cells were compared with the treated cells to determine the migration of the cells. The photomicrographs of the cells were recorded under different magnifications utilizing Zeiss microscope with attached $35 \mathrm{~mm}$ camera.

Clonogenic assay: A soft-agar sandwitch assay was performed. Cells were sandwiched between $0.6 \%$ and $0.3 \%$ agarose in DMEM medium containing 5\% FBS in a six-well chamber (500 cells/chamber), and treated with buffer (Control), or respective agent for noted time and dose at $37^{\circ} \mathrm{C}$ humidified $\mathrm{CO}_{2}$ incubator. The colonies from multiple random fields were counted, compared to control and photographed essentially as described before [20-22].

\section{Three-dimensional NSCLC sphere assays}

The parental and TKI-resistant NSCLC cells from a two-dimensional culture plate with $\sim 70-80 \%$ confluence were utilized for $3 \mathrm{D}$ assays. We performed the three- 
dimensional NSCLC sphere cultures by essentially following the methods described by us before $[22,22]$. Briefly, the cells were washed twice in $1 \times$ PBS and trypsinized following established protocols. We then pelleted the cells at $200 \times \mathrm{g}$ at room temperature, and re-suspended them in $5 \mathrm{ml}$ of sphere media (DMEM/ F12 supplemented with $2 \mathrm{mM}$ L-glutamine, $100 \mathrm{U} / \mathrm{ml}$ penicillin, $100 \mathrm{U} / \mathrm{ml}$ streptomycin, $1 \times$ B27 supplement, $20 \mathrm{ng} / \mathrm{ml}$ recombinant human epidermal growth factor (EGF; Sigma), and $10 \mathrm{ng} / \mathrm{ml}$ recombinant human basic fibroblast growth factor (bFGF; R\&D Systems). We seeded $\sim 5000$ viable cells per $\mathrm{ml}$ in an ultra-low adherent $60 \mathrm{~mm}$ plate and incubated them at $37^{\circ} \mathrm{C}$ and $5 \% \mathrm{CO}_{2}$ for two weeks without disturbing the plates. After the spheres formed, we added fresh media with or without $10 \mu \mathrm{M}$ CFM-4.16 and continued incubating cells for additional $24 \mathrm{~h}$ at $37^{\circ} \mathrm{C}$ and $5 \% \mathrm{CO}_{2}$. At the end of the incubation period, we photographed the spheres in the untreated and treated plates as described [48].

\section{Establishment of NSCLC cell-derived xenografts in immunocompromised mice}

The experiments involving generation of Rociletinib-resistant H1975 NSCLC cell-derived subcutaneous xenografts were performed according to our previously published methods and protocols approved by the Institutional Laboratory Animal care \& Use Committees at the Florida A\&M University [20, 21]. Female, 4-6-weeks old (20-25 g) Balb/c nude mice were purchased from Charles River Laboratories (Horsham, PA, USA). Following suitable acclimation of animals, Five million H1975 (rocelitinib-resistant) cells were suspended in Hanks Balanced salt solution and implanted s.c. in right flanks of the nude mice using a 27-gauge needle. Tumors were allowed to grow for 15 days and when tumors became palpable $(200 \mathrm{~mm} 3)$, the mice were randomly assigned to treatment or control groups with 6 animals in each group. The treatment groups consisted of control, CFM-4.16 NLF (50 mg/kg), Sorafenib (30 mg/kg), and CFM-4.16 NLF plus Sorafenib every alternate day for 14 days. All preparations were given by oral gavage. The mice were followed for their tumor burden and mobility for the next three weeks after which the experiment was terminated and the mice were sacrificed. Tumor tissues were collected immediately after tumor volume measurement. Tumor volume was calculated by the modified ellipsoidal formula: Tumor volume $=1 / 2$ (length $\times$ width2) Representative tumor samples were stored at $-80^{\circ} \mathrm{C}$ and in $10 \%$ formalin for subsequent analysis.

\section{Statistical analysis}

The statistical analysis was done using Prism 6.0 software (Graph Pad Software Inc., San Diego, CA, USA). The data were expressed as mean \pm SEM and analyzed using a two-tailed Student $t$-test or one-way ANOVA followed by a post hoc test. A $p$ value of $<0.05$ was considered statistically significant.

\section{Author contributions}

AKR, MS, AKI, and GB conceived and designed the experiments; VTC, HA, SS, JV, AM, IV, SS, MM, LAP, and EL performed the experiments; VTC, HA, and SS analyzed data; and AKR wrote the manuscript.

\section{ACKNOWLEDGMENTS}

The authors thankfully acknowledge W. Marston Linehan, M.D., the UOB Tumor Cell Line Repository, National Cancer Institute at Bethesda, Maryland, for providing UOK262 and UOK268 HLRCC cells.

\section{CONFLICTS OF INTEREST}

None.

\section{GRANT SUPPORT}

This work was supported by the Department of Veterans Affairs Merit Review grant (AKR), The NIH RO1 CA129343 (GB), The Wayne State University startup grant (AKI), the NCI/NIH Cancer Center Support Grant to Karmanos Cancer Institute, the National Institutes of Health's Minority Biomedical Research Support (MBRS)-SC1 program grant \# SC1 GM092779-01 (MS), NCI grant \# R21CA175618 (MS), and National Institute on Minority Health and Health Disparities (NIMHD) P20 program grant \#1P20 MD006738-03 (MS).

\section{REFERENCES}

1. Siegel R, Miller K, Jemal A. Cancer statistics 2016. CA: Cancer J Clin. 2016; 66:7-30.

2. Howlader N, Noone AM, Krapcho M, Miller D, Bishop K, Altekruse SF, Kosary CL, Yu M, Ruhl J, Tatalovich Z, Mariotto A, Lewis DR, Chen HS, et al. SEER Cancer Statistics Review, 1975-2013, National Cancer Institute. Bethesda, MD.

3. Horn L, Eisenberg R, Gius D, Kimmelshue KN, Massion PP, Putnam JB, Robinson CG, Carbone DP. Cancer of the lung: Non-small cell lung cancer and small cell lung cancer. In: Niederhuber JE, Armitage JO, Doroshow JH, Kastan MB, Tepper JE, editors. Abeloff's Clinical Oncology. 5th ed. Philadelphia (Pa): Elsevier; 2014; 1143-92.

4. Wang X, Goldstein D, Crowe PJ, Yang JL. Next-generation EGFR/HER tyrosine kinase inhibitors for the treatment of patients with non-small-cell lung cancer harboring EGFR mutations: a review of the evidence. Onco Targets Ther. 2016; 9:5461-73. 
5. Planchard D, Loriot Y, André F, Gobert A, Auger N, Lacroix L, Soria JC. EGFR-independent mechanisms of acquired resistance to AZD9291 in EGFR T790M-positive NSCLC patients. Ann Oncol. 2015; 26:2073-8.

6. Chabon JJ, Simmons AD, Lovejoy AF, Esfahani MS, Newman AM, Haringsma HJ, Kurtz DM, Stehr H, Scherer F, Karlovich CA, Harding TC, Durkin KA, Otterson GA, et al. Circulating tumour DNA profiling reveals heterogeneity of EGFR inhibitor resistance mechanisms in lung cancer patients. Nat Commun. 2016; 7:11815.

7. Rishi AK, Zhang L, Boyanapalli M, Wali A, Mohammad RM, Yu Y, Fontana JA, Hatfield JS, Dawson MI, Majumdar AP, Reichert U. Identification and characterization of a cell cycle and apoptosis regulatory protein-1 as a novel mediator of apoptosis signaling by retinoid CD437. J Biol Chem. 2003; 278:33422-35.

8. Rishi AK, Zhang L, Yu Y, Jiang Y, Nautiyal J, Wali A, Fontana JA, Levi E, Majumdar AP. Cell cycle and apoptosis regulatory protein [CARP]-1 is involved in apoptosis signaling by epidermal growth factor receptor. J Biol Chem. 2006; 281:13188-98.

9. Puliyappadamba VT, Wu W, Bevis D, Zhang L, Polin L, Kilkuskie R, Finley RL Jr, Larsen SD, Levi E, Miller FR, Wali A, Rishi AK. Antagonists of Anaphase-promoting Complex (APC)-2-Cell Cycle and Apoptosis Regulatory Protein (CARP)-1 Interaction Are Novel Regulators of Cell Growth and Apoptosis. J Biol Chem. 2011; 286:38000-38017.

10. Muthu M, Cheriyan VT, Rishi AK. CARP-1 / CCAR1: A biphasic regulator of cancer cell growth and apoptosis. Oncotarget. 2015; 6:6499-6510. https://doi.org/10.18632/ oncotarget.3376.

11. Kim JH, Yang CK, Heo K, Roeder RG, An W, Stallcup MR. CCAR1, a key regulator of mediator complex recruitment to nuclear receptor transcription complexes. Mol Cell. 2008; 31:510-519.

12. Ou CY, Chen TC, Lee JV, Wang JC, Stallcup MR. Coregulator CCAR1 positively regulates adipocyte differentiation through the glucocorticoid signaling pathway. J Biol Chem. 2014; 289:17078-17086.

13. Harper JW, Burton JL, Solomon MJ. The anaphasepromoting complex: it's not just for mitosis any more. Genes Dev. 2002; 16:2179-206.

14. Lehman NL, Tibshirani R, Hsu JY, Natkunam Y, Harris BT, West RB, Masek MA, Montgomery K, van de Rijn M, Jackson PK. Oncogenic regulators and substrates of the anaphase promoting complex/cyclosome are frequently overexpressed in malignant tumors. Am J Pathol. 2007; 170:1793-805.

15. Zhang J, Wan L, Dai X, Sun Y, Wei W. Functional characterization of Anaphase Promoting Complex/ Cyclosome (APC/C) E3 ubiquitin ligases in tumorigenesis. Biochim Biophys Acta. 2014; 1845:277-293.
16. Yoo AS, Bais C, Greenwald I. Crosstalk between the EGFR and LIN-12/Notch pathways in C. elegans vulval development. Science. 2004; 303:663-666.

17. Jamal S, Cheriyan VT, Muthu M, Munie S, Levi E, Ashour AE, Pass HI, Wali A, Singh M, Rishi AK. CARP-1 functional mimetics are a novel class of small molecule inhibitors of malignant pleural mesothelioma cells. PLoS One. 2014; 9:e89146.

18. Muthu M, Cheriyan VT, Munie S, Levi E, Frank J, Ashour AE, Singh M, Rishi AK. Mechanisms of neuroblastoma cell growth inhibition by CARP-1 functional mimetics. PLoS One. 2014; 9:e102567.

19. Ashour AE, Jamal S, Cheryan VT, Muthu M, Zoheir KMA, Alafeefy AM, Abd-Allah AR, Levi E, Tarca AL, Polin LA, Rishi AK. CARP-1 functional mimetics: A novel class of small molecule inhibitors of medulloblastoma cell growth. PLoS One. 2013; 8:e66733.

20. Muthu M, Somagoni J, Cheriyan VT, Munie S, Levi E, Ashour AE, Yassin AE, Alafeefy AM, Sochacki P, Polin LA, Reddy KB, Larsen SD, Singh M, Rishi AK. Identification and testing of novel CARP-1 functional mimetic compounds as inhibitors of non-small cell lung and triple negative breast cancers. J Biomed Nanotechnol. 2015; 11:1608-1627.

21. Cheriyan VT, Muthu M, Patel K, Sekhar S, Rajeswaran W, Larsen SD, Polin L, Levi E, Singh M, Rishi AK. CARP-1 functional mimetics are novel inhibitors of drug-resistant triple negative breast cancers. Oncotarget. 2016; 7: 73370-88. https://doi.org/10.18632/oncotarget.12333.

22. Cheriyan VT, Alsaab HO, Sekhar S, Stieber C, Kesharwani P, Sau S, Muthu M, Polin LA, Levi E, Iyer AK, Rishi AK. A CARP-1 functional mimetic loaded vitamin E-TPGS micellar nano-formulation for inhibition of renal cell carcinoma. Oncotarget. 2017; 8:104928-45. https://doi.org/10.18632/oncotarget.20650.

23. Rosenzweig SA. Acquired resistance to drugs targeting receptor tyrosine kinases. Biochem Pharmacol. 2012; 83:1041-48.

24. Huang L, Fu L. Mechanisms of resistance to EGFR tyrosine kinase inhibitors. Acta Pharm Sin B. 2015; 5:390-401.

25. Botting GM, Rastogi I, Chhabra G, Nlend M, Puri N. Mechanism of Resistance and Novel Targets Mediating Resistance to EGFR and c-Met Tyrosine Kinase Inhibitors in Non-Small Cell Lung Cancer. PLoS One. 2016; 10:e0136155.

26. Shien K, Papadimitrakopoulou VA, Ruder D, Behrens C, Shen L, Kalhor N, Song J, Lee JJ, Wang J, Tang X, Herbst RS, Toyooka S, Girard L, et al. JAK1/STAT3 activation through a proinflammatory cytokine pathway leads to resistance to molecularly targeted therapy in non-small cell lung cancer. Mol Cancer Ther. 2017; 16:2234-2245.

27. Harada D, Takigawa N, Kiura K. The Role of STAT3 in NonSmall Cell Lung Cancer. Cancers (Basel). 2014; 6:708-722. 
28. Kawakami H, Okamoto I, Okamoto W, Tanizaki J, Nakagawa K, Nishio K. Targeting MET Amplification as a New Oncogenic Driver. Cancers. 2014; 6:1540-1552.

29. Piotrowska Z, Niederst MJ, Karlovich CA, Wakelee HA, Neal JW, Mino-Kenudson M, Fulton L, Hata AN, Lockerman EL, Kalsy A, Digumarthy S, Muzikansky A, Raponi M, et al. Heterogeneity Underlies the Emergence of EGFR T790 Wild-Type Clones Following Treatment of T790M-Positive Cancers with a Third Generation EGFR Inhibitor. Cancer Discov. 2015; 5:713-22.

30. Minari R, Bordi P, Tiseo M. Third-generation epidermal growth factor receptor-tyrosine kinase inhibitors in T790Mpositive non-small cell lung cancer: review on emerged mechanisms of resistance. Transl Lung Cancer Res. 2016; 5:695-708. https://doi.org/10.21037/tlcr.2016.12.02.

31. Gnoni A, Marech I, Silvestris N, Vacca A, Lorusso V. Dasatinib: an anti-tumour agent via Src inhibition. Curr Drug Targets. 2011; 12:563-78.

32. Scagliotti G, von Pawel J, Novello S, Ramlau R, Favaretto A, Barlesi F, Akerley W, Orlov S, Santoro A, Spigel D, Hirsh V, Shepherd FA, Sequist LV, et al. Phase III multinational, randomized, double-blind, placebocontrolled study of Tivantinib (ARQ 197) plus erlotinib versus erlotinib alone in previously treated patients with locally advanced or metastatic nonsquamous non-small-cell lung cancer. J Clin Oncol. 2015; 33:2667-2674.

33. Kyriakis JM, Avruch J. Mammalian MAPK signal transduction pathways activated by stress and inflammation: A 10-year update. Physiol Rev. 2012; 92:689-737.

34. Koul HK, Pal M, Koul S. Role of p38 MAP Kinase Signal Transduction in Solid Tumors. Genes Cancer. 2013; 4: 342-59. https://doi.org/10.1177/1947601913507951.

35. Genovese MC. Inhibition of $\mathrm{p} 38$ : has the fat lady sung? Arthritis Rheum. 2009; 60:317-20.

36. Walker AJ, Wedam S, Amiri-Kordestani L, Bloomquist E, Tang S, Sridhara R, Chen W, Palmby TR, Fourie Zirkelbach J, Fu W, Liu Q, Tilley A, Kim G, et al. FDA approval of palbociclib in combination with fulvestrant for the treatment of hormone receptor-positive, HER2-negative metastatic breast cancer. Clin Cancer Res. 2016; 22:4968-4972.

37. Kim G, Ison G, McKee AE, Zhang H, Tang S, Gwise T, Sridhara R, Lee E, Tzou A, Philip R, Chiu HJ, Ricks TK, Palmby T, et al. FDA approval summary: Olaparib monotherapy in patients with deleterious germline BRCAmutated advanced ovarian cancer treated with three or more lines of chemotherapy. Clin Cancer Res. 2015; 21:4257-61.

38. Noeparast A, Teugels E, Giron P, Verschelden G, De Brakeleer S, Decoster L, De Grève J. Non-V600 BRAF mutations recurrently found in lung cancer predict sensitivity to the combination of Trametinib and Dabrafenib. Oncotarget. 2016; 8:60094-60108. https://doi.org/10.18632/oncotarget.11635.

39. Smalley KS, Xiao M, Villanueva J, Nguyen TK, Flaherty KT, Letrero R, Van Belle P, Elder DE, Wang Y, Nathanson KL, Herlyn M. CRAF inhibition induces apoptosis in melanoma cells with non-V600E BRAF mutations. Oncogene. 2009; 28:85-94.

40. Wilhelm SM, Adnane L, Newell P, Villanueva A, Llovet JM, Lynch M. Preclinical overview of sorafenib, a multikinase inhibitor that targets both Raf and VEGF and PDGF receptor tyrosine kinase signaling. Mol. Cancer Ther. 2008; 7:3129-40.

41. Johnson DB, Flaherty KT, Weber JS, Infante JR, Kim KB, Kefford RF, Hamid O, Schuchter L, Cebon J, Sharfman WH, McWilliams RR, Sznol M, Lawrence DP, et al. Combined BRAF (Dabrafenib) and MEK inhibition (Trametinib) in patients with BRAFV600-mutant melanoma experiencing progression with single-agent BRAF inhibitor. J Clin Oncol. 2014; 32:3697-704.

42. Flaherty KT, Infante JR, Daud A, Gonzalez R, Kefford RF, Sosman J, Hamid O, Schuchter L, Cebon J, Ibrahim N, Kudchadkar R, Burris HA 3rd, Falchook G, et al. Combined BRAF and MEK Inhibition in Melanoma with BRAF V600 Mutations. N Engl J Med. 2012; 367:1694-703.

43. Zhou J, Chen Z, Malysa A, Li X, Oliveira P, Zhang Y, Bepler G. A kinome screen identifies checkpoint kinase 1 (CHK1) as a sensitizer for RRM1-dependent gemcitabine efficacy. PLoS One. 2013; 8:e58091.

44. Chou TC, Talalay P. Quantitative analysis of dose-effect relationships: the combined effects of multiple drugs or enzyme inhibitors. Adv Enzyme Regul. 1984; 22:27-55.

45. Chou TC. Theoretical basis, experimental design, and computerized simulation of synergism and antagonism in drug combination studies. Pharmacol Rev. 2006; 58:621-681.

46. Zhang N, Wu ZM, McGowan E, Shi J, Hong ZB, Ding $\mathrm{CW}, \mathrm{Xia} \mathrm{P}, \mathrm{Di} \mathrm{W}$. Arsenic trioxide and cisplatin synergism increase cytotoxicity in human ovarian cancer cells: therapeutic potential for ovarian cancer. Cancer Sci. 2009; 100:2459-2464.

47. Chou TC, Martin N. CompuSyn Software for Drug Combinations and for General Dose-Effect Analysis, and User's Guide. Paramus (NJ): ComboSyn; 2007.

48. Lombardo Y, de Giorgio A, Coombes CR, Stebbing J, Castellano L. Mammosphere Formation Assay from Human Breast Cancer Tissues and Cell Lines. J Vis Exp. 2015; 97:e52671. https://doi.org/10.3791/52671. 